\title{
Cointegration in Fractional Systems with Deterministic Trends
}

\author{
by \\ Peter M Robinson and Fabrizio lacone \\ London School of Economics and Political Science
}

Contents:

Abstract

1. Introduction

2. Least Squares Estimates of Model I

3. Least Squares Estimates of Model II

4. Generalized Least Squares Estimates of Model III

5. Monte Carlo Simulations

6. Empirical Analysis of the PPP Hypothesis

Appendix

References

Tables $1-11$

Figures $1-4$

Discussion Paper

No.EM/04/476

May 2004
The Suntory Centre

Suntory and Toyota International Centres for Economics and Related Disciplines

London School of Economics and Political Science Houghton Street

London WC2A $2 \mathrm{AE}$

Tel.: 02079556698

* This research was supported by ESRC Grants R000235892 and R000239936. We thank two referees for constructrive comments. 


\section{Abstract}

We consider a cointegrated system generated by processes that may be fractionally integrated, and by additive polynomial and generalized polynomial trends. In view of the consequent competition between stochastic and deterministic trends, we consider various estimates of the cointegrating vector and develop relevant asymptotic theory, including the situation where fractional orders of integration are unknown.

Keywords: Fractional cointegration; deterministic trends; ordinary least squares estimation; generalized least squares estimation; Wald tests.

JEL Nos.: C22

(C) by Peter M Robinson. All rights reserved. Short sections of text, not to exceed two paragraphs, may be quoted without special permission provided that full credit, including $\odot$ notice, is given to the source.

Contact address: Professor P M Robinson, Department of Economics, London School of Economics and Political Science, Houghton Street, London WC2A 2AE, UK. Email: p.m.robinson@Ise.ac.uk 


\section{Introduction}

Fractional cointegration analysis has developed on the basis that cointegrating relationships between nonstationary economic variables may exist without observable processes necessarily being unit root $(I(1))$ processes or cointegrating errors necessarily being short memory $(I(0))$ processes. Optimal rules of statistical inference based on the $I(1) / I(0)$ prescription lose their most desirable properties when the integration orders on which they are based are misspecified, while on the other hand methodology developed in the $I(1) / I(0)$ setting is not designed to detect fractional cointegration. A fractional cointegration analysis that allows integration orders to be unknown, and nests integer-valued orders as special cases, thus affords a substantial increase in flexibility. Though the fractional cointegration literature is dwarfed by the $I(1) / I(0)$ literature, there are now a number of references dealing with theoretical issues (see e.g. Dolado and Marmol (1997), Chan and Terrin (1995), Jeganathan (1999), Kim and Phillips (2000), Velasco (2000), Hassler, Marmol and Velasco (2002)) and empirical applications (see e.g. Cheung and Lai (1993)).

The large $I(1) / I(0)$ literature focusses on many features of economic time series, in particular recognizing empirical evidence that the stochastic unit root trend frequently needs to be supplemented by a deterministic trend, such as one increasing linearly with time (see e.g. West (1988), Stock and Watson (1988), Johansen (1991), Hansen (1992), Perron and Campbell (1993), Campos et al (1996)). For empirical applications to the analysis of demand for money, see Hoffman and Rasche (1991), Stock and Watson (1993), and to the PPP/UIP relations see Johansen and Juselius (1992). A review of applications of cointegrated models with a background in economic theory is in Söderlind and Vredin (1996), and a particular treatment of cotrending, deterministic and stochastic cointegration is in Ogaki and Park (1997), who modelled the allocation of income in consumption of durable and non-durable goods.

On the other hand the fractional cointegration literature has mostly not allowed for deterministic trends. For example, this is the case in Robinson and Marinucci (2001) (hereafter RM) (see also Robinson and Marinucci, 2003) who study limiting behaviour of least squares and narrow-band least squares estimates of cointegrating coefficients, and in Robinson and Hualde (2003) (hereafter RH), who provide optimal methods for estimating a bivariate, purely stochastic, cointegrated system that allows for unknown integration orders and very general parametric modelling of stationary short memory, and show that Wald tests on the cointegrating coefficient have the asymptotic null $\chi^{2}$ limit distribution found by, e.g., Phillips and Hansen (1990), Phillips (1991a,b), Johansen (1991), Hansen (1992) in the $I(1) / I(0)$ case. The present paper develops properties of estimates of the cointegrating coefficient in a bivariate model that either ignore or take account of additive deterministic trends.

To introduce some formal definitions, define the difference operator $\Delta=$ 
$1-L$, where $L$ is the lag operator, and, for any real $d, d \neq-1,-2, .$. ,

$$
(1-z)^{-d}=\sum_{j=0}^{\infty} a_{j}(d) z^{j}, \quad a_{j}(d)=\frac{\Gamma(j+d)}{\Gamma(d) \Gamma(j+1)},
$$

with $\Gamma$ denoting the gamma function. Denoting by $v_{t}$ a covariance stationary process with zero mean and spectral density that is bounded and bounded away from zero at all frequencies, for any real-valued $d$ we call $w_{t}$ an $I(d)$ (stochastic) sequence if

$$
w_{t}=\Delta^{-d} v_{t}^{\#},
$$

where, throughout, the \# superscript has the meaning

$$
v_{t}^{\#}=v_{t} 1(t>0),
$$

where $1(\cdot)$ is the indicator function. Thus (3) implies that $w_{t}=0, t \leq 0$, so that even within the 'stationarity' region for $d, d<1 / 2, w_{t}$ is actually not covariance stationary, though it may be thought of as 'asymptotically stationary' for such $d$. The truncation in (2) is introduced to cater for 'nonstationary' values, $d \geq 1 / 2$, where $w_{t}$ would otherwise blow up.

We also introduce a corresponding class of deterministic sequences. We say $w_{t}$ is a $J(d)$ (deterministic) sequence if

$$
w_{t} \sim \frac{t^{d-1 / 2}}{\Gamma(d+1 / 2)}, \text { as } t \rightarrow \infty,\left|w_{t}-w_{t+1}\right| \leq C w_{t} / t, t \geq 1,
$$

where throughout $C$ denotes a finite generic constant and $\sim$ indicates that the ratio between left- and right- sides tends to a finite positive constant. The definitions of $I(d)$ and $J(d)$ match in the sense that:

(i) If $w_{t}$ is $I(d)$ then $\Delta^{c} w_{t}^{\#}$ is $I(d-c)$; if $w_{t}$ is $J(d)$ then $\Delta^{c} w_{t}^{\#}$ is $J(d-c)$.

(ii) If $d>0$, then: if $w_{t}$ is $I(d), E w_{t}^{2} \sim t^{2 d-1}$ as $t \rightarrow \infty$; if $w_{t}$ is $J(d)$, $w_{t}^{2} \sim t^{2 d-1}$ as $t \rightarrow \infty$.

We expect then, in a system containing $I(d)$ and $J(e)$ sequences, that (fractional) differencing has a similar effect on both and that there will be a tendency for an $I(d)$ component to dominate a $J(e)$ one for $d>e$, and vice versa when $d<e$. This is most simply seen in an additive model. We consider a bivariate observable sequence $\left(x_{t}, y_{t}\right)$ given by the components model

$$
\begin{aligned}
x_{t} & =g_{1 t}\left(\phi_{1}\right)+h_{1 t}\left(\delta_{1}\right), t \geq 1, \\
y_{t} & =g_{2 t}\left(\phi_{2}\right)+h_{2 t}\left(\delta_{2}\right), t \geq 1, \\
g_{1 t}\left(\phi_{1}\right) & =h_{1 t}\left(\delta_{1}\right)=g_{2 t}\left(\phi_{2}\right)=h_{2 t}\left(\delta_{2}\right) \equiv 0, t \leq 0,
\end{aligned}
$$

where $g_{i t}(d)$ is $J(d)$ and $h_{i t}(d)$ is $I(d), i=1,2$ and the $g_{i t}\left(\phi_{i}\right), h_{i t}\left(\delta_{i}\right)$ are unobservable sequences. Robinson and Marinucci (2000) discussed a fractional model of form (5)-(7). In particular they considered the asymptotic behaviour of 
the sample covariance matrix of $\left(x_{t}, y_{t}\right)$, based on observations at $t=1,2, \ldots, n$, and of the averaged periodogram of $\left(x_{t}, y_{t}\right)$, being the normalized sum of the periodogram matrix of $\left(x_{t}, y_{t}\right)$ over $m$ Fourier frequencies close to the origin, namely $\lambda_{j}=2 \pi j / n, j=1,2, \ldots, m$, where $1 / m+m / n \rightarrow 0$ as $n \rightarrow \infty$; their stress is on cases where $\phi_{i}>0, \delta_{i}>1 / 2, i=1,2$, when the sample covariance matrix and the averaged periodogram have the same asymptotic behaviour (since they are equal when $m=n$, and low frequency components dominate the sample covariance matrix). Robinson and Marinucci (2000) described how stochastic or deterministic components dominate, depending on the values of the $\phi_{i}, \delta_{i}$. They also considered a (narrow-band) regression of $y_{t}$ on $x_{t}$, in case the deterministic component of $x_{t}$ dominates the error, and the stochastic component of the error dominates its deterministic component.

Regression relations also arise in case of cointegration. However, whereas in the case of Robinson and Marinucci (2000) the estimate of the regression coefficient is consistent and asymptotically normal due to the dominating effect of the deterministic component of $x_{t}$, consistency in cointegrated systems involving no deterministic components is due to the domination of the stochastic component of the cointegrating error by that of the regressor. Here we look at implications of deterministic components. On the basis of (5)-(7), it is supposed that $\delta_{1}=\delta_{2}=\delta$ and there exists $\nu \neq 0$ such that $h_{2 t}(\delta)-\nu h_{1 t}(\delta)$ is $I(\gamma), \gamma<\delta$.

Irrespective of whether or not we commence from the component model (5)(7), we assume the following cointegrated system as data generating mechanism,

$$
\begin{aligned}
& y_{t}=\nu x_{t}+\sum_{j=1}^{p_{1}} \mu_{1 j} t^{\phi_{1 j}-1 / 2}+u_{1 t}(-\gamma), \\
& x_{t}=\sum_{j=1}^{p_{2}} \mu_{2 j} t^{\phi_{2 j}-1 / 2}+u_{2 t}(-\delta),
\end{aligned}
$$

where for any sequence $\left\{v_{t}\right\}$, and any $c$, we use the notation of $\mathrm{RH}$,

$$
v_{t}(c)=\Delta^{c} v_{t}^{\#} .
$$

In (8), $(9), u_{t}=\left(u_{1 t}, u_{2 t}\right)^{\prime}$, with the prime denoting transposition, is a jointly covariance stationary process with zero mean and spectral density matrix, $f(\lambda)$, satisfying

$$
E\left(u_{0} u_{j}^{\prime}\right)=\int_{-\pi}^{\pi} e^{i j \lambda} f(\lambda) d \lambda
$$

that is nonsingular and continuous at all frequencies;

$$
\begin{aligned}
\nu & \neq 0, \\
\delta & >\max \left(\gamma, \frac{1}{2}\right) ;
\end{aligned}
$$

the $\phi_{i j}$ are real numbers satisfying

$$
\begin{aligned}
& \phi_{11}>\ldots>\phi_{1 p_{1}}>0, \\
& \phi_{21}>\ldots>\phi_{2 p_{2}}>0,
\end{aligned}
$$


and also, for $0 \leq p_{11} \leq p_{1}, 0 \leq p_{21} \leq p_{2}$,

$$
\begin{aligned}
& \phi_{1 p_{11}}>\gamma, \phi_{1, p_{11}+1}<\gamma, \\
& \phi_{2 p_{21}}>\delta, \phi_{2, p_{21}+1}<\delta ;
\end{aligned}
$$

for $p_{11}=p_{1} \quad\left(p_{21}=p_{2}\right)$, the quantities $p_{11}+1\left(p_{21}+1\right)$ are not defined, so the second inequality of $(16)((17))$ is irrelevant, while for $p_{11}=0\left(p_{21}=0\right)$ all $\phi_{1 j}$ $\left(\phi_{2 j}\right)$ are less than $\gamma(\delta)$, so the first inequality of $(16)((17))$ is irrelevant. In (8), (9) an intercept term appears when $\phi_{1 j}=1 / 2, \phi_{2 j}=1 / 2$, respectively, while integer powers are also possible, but we allow for the $\phi_{i j}$ to be any real values satisfying (14)-(17).

The convention that powers of $t$ be denoted $\phi_{i j}-1 / 2$ rather than $\phi_{i j}$ is to enable convenient comparison with integration orders, as indicated by our definitions of $I(d)$ and $J(d)$ sequences. It is possible that one or more of the $\mu_{i j}$ are actually zero, though we do not know this, and we define

$$
j^{\dagger}=\min \left\{j: \mu_{1 j} \neq 0\right\}, \quad j^{\ddagger}=\min \left\{j: \mu_{2 j} \neq 0\right\} .
$$

We allow for this possibility because we wish (8), (9) to nest the working model used in estimation, and it is possible that one or more regressors $t^{\phi_{i j}-1 / 2}$ will be included in the latter whose coefficient is zero. For brevity write $\phi_{1 \dagger}=\phi_{1 j^{\dagger}}$, $\phi_{2 \ddagger}=\phi_{2 j^{\ddagger}}, \mu_{1 \dagger}=\mu_{1 j^{\dagger}}, \mu_{2 \ddagger}=\mu_{2 j^{\ddagger}}$.

The truncations in (8), (9) imply that $x_{t}=y_{t}=0, t \leq 0$, and that $x_{t}, y_{t}$ $t \geq 1$, have finite variance. Without truncation they would not be well-defined in mean square, since $\delta>1 / 2$, while $\Delta^{-\gamma} u_{1 t}$ is not well-defined in mean square when $\gamma \geq 1 / 2$. In particular, $x_{t}$, and thus $y_{t}$, have variance that increases with $t$ (like $\left.t^{2 \delta-1}\right)$. Note that the elements of $u_{t}^{\#}$ are $I(0)$ processes, while $u_{1 t}(-\gamma)$ is an $I(\gamma)$ process and $u_{2 t}(-\delta)$ is an $I(\delta)$ process.

Define, for $i=1,2$, the $p_{i 1} \times 1$ vector sequences

$$
\begin{aligned}
g_{i}(t) & =\left(t^{\phi_{i 1}-1 / 2}, \ldots, t^{\phi_{i p_{i 1}}-1 / 2}\right)^{\prime}, t \geq 1, \\
& =(0, \ldots, 0)^{\prime}, t \leq 0
\end{aligned}
$$

where the prime denotes transposition, and denote by $\mu_{i}, i=1,2$, the $p_{i 1} \times 1$ vectors whose $j$ th elements are respectively $\mu_{i j}, i=1,2$. In view of (18), (19) note that $\mu_{1}^{\prime} g_{1}(t)$ is a $J\left(\phi_{1 \dagger}\right)$ sequence and $\mu_{2}^{\prime} g_{2}(t)$ is a $J\left(\phi_{2 \ddagger}\right)$ sequence.

The working models that are estimated are as follows.

Working Model I This is

$$
y_{t}=\nu x_{t}+v_{t},
$$

where $\nu$ is estimated by non-intercept OLS, as if the errors $v_{t}$ were serially uncorrelated random variables orthogonal to $x_{t}$. The main issues here are the effect of the misspecification error caused by neglecting the deterministic component in (8) and simultaneous equation bias due to (9). 
Working Model II This is

$$
y_{t}=\nu x_{t}+\mu_{1}^{\prime} g_{1}(t)+v_{t},
$$

where $\nu$ and $\mu_{1}$ are simultaneously estimated by multiple OLS, again as if the errors $v_{t}$ were serially uncorrelated random variables orthogonal to $x_{t}$. Here, one issue is the robustness to possible omission of the component $s_{1 t}=$ $\sum_{j=p_{11}+1}^{p_{1}} \mu_{1 j} t^{\phi_{1 j}-1 / 2}$. Indeed the $\mu_{1 j}, p_{11}+1 \leq j \leq p_{1}$, are not consistently estimable. Another is the simultaneous equations bias due to (9) and error autocorrelation due to (8).

\section{Working Model III This is}

$$
\begin{aligned}
& y_{t}=\nu x_{t}+\mu_{1}^{\prime} g_{1}(t)+v_{1 t}, \\
& x_{t}=\mu_{2}^{\prime} g_{2}(t)+v_{2 t}
\end{aligned}
$$

where $\nu, \mu_{1}$ and $\mu_{2}$ are simultaneously estimated by a form of generalized least squares (GLS) as if $v_{1 t}=u_{1 t}(-\gamma), v_{2 t}=u_{2 t}(-\delta)$, properly accounting for both autocorrelation and simultaneity, and with either $\gamma$ and/or $\delta$ assumed known or suitably estimated in a side calculation. For our discussion of GLS we assume also that

$$
\beta>\frac{1}{2},
$$

where $\beta=\delta-\gamma$. The requirement (25) includes the usual case of $I(0)$ cointegrating errors and $I(1) x_{t}$; the case $\beta \leq \frac{1}{2}$ leads to quite different asymptotics, see e.g. Jeganathan (1999), Hualde and Robinson (2001). The GLS method is an extension of that of $\mathrm{RH}$, in which $\mu_{1}=0, \mu_{2}=0$ was correctly assumed a priori. Comparison with (8), (9) indicates that in fact $v_{1 t}=s_{1 t}+u_{1 t}(-\gamma)$, $v_{2 t}=s_{2 t}+u_{2 t}(-\delta)$, where $s_{2 t}=\sum_{j=p_{21}+1}^{p_{2}} \mu_{2 j} t^{\phi_{2 j}-1 / 2}$, so that we have to show that ignoring $s_{1 t}, s_{2 t}$ has no asymptotic effect; none of the $\mu_{i j}$ in these is consistently estimable. Thus robustness of RH's estimates to omission of such $s_{i t}$ will be implied.

We could replace the deterministic terms in (8), (9) by more general ones, and in particular could specify $s_{1 t}$ and $s_{2 t}$ in terms of bounds, rather than precise quantities, but we prefer the simple set-up afforded by (8), (9). While we allow for non-integer powers $\phi_{i j}-1 / 2$, this is not on the basis of arguing that these are likely to be of great practical value (though they may turn out to provide improved approximations to some data), but rather because it affords a precise treatment of the competition between stochastic and deterministic trends. Indeed, though we mostly regard $\gamma$ and $\delta$ as unknown, the $\phi_{i j}$ in $g_{1}(t)$ and $g_{2}(t)$, when these are included, are assumed known; there are difficulties with asymptotic theory for, say, OLS estimation of the $\phi_{i j}$ due to lack of uniform convergence of the objective function.

In the following three sections we discuss rates of convergence (if any) and asymptotic distributional properties of the estimates of working models I, II and 
III respectively. Proof details are left to appendices. Section 5 contains a small Monte Carlo study of finite sample performance, and Section 6 an empirical application to testing the PPP hypothesis on the basis of data for three US cities.

\section{LEAST SQUARES ESTIMATES OF MODEL I}

The OLS estimate of $\nu$ in working Model I (21) is

$$
\bar{\nu}=M_{x y} / M_{x x},
$$

where for any column vector or scalar sequences $a_{t}, b_{t}, M_{a b}=\sum_{t=1}^{n} a_{t} b_{t}^{\prime}$, and, throughout the paper, $\sum_{t}$ denotes $\sum_{t=1}^{n}$. The estimate $\bar{\nu}$ has the advantage of not requiring knowledge of $\delta$ and/or $\beta$. Under (8), (9) with $\mu_{i j}=0$ for all $i, j$, RM showed under mild additional conditions that $\bar{\nu}$ is $n^{\min (2 \delta-1, \beta)}$ consistent (except when both $\delta>\beta$ and $2 \delta-\beta=1$ hold, in which case it is $\left(n^{\beta} / \log n\right)$-consistent).

Denote by $I_{m}$ the $m$-rowed identity matrix. We introduce

Assumption 1. The process $u_{t}, t=0, \pm 1, \ldots$, has representation

$$
u_{t}=A(L) \varepsilon_{t},
$$

where

$$
A(s)=I_{2}+\sum_{j=1}^{\infty} A_{j} s^{j},
$$

and the $A_{j}$ are $2 \times 2$ matrices such that:

(i)

$$
\operatorname{det}\{A(s)\} \neq 0,|s|=1 ;
$$

(ii) $A\left(e^{i \lambda}\right)$ is differentiable in $\lambda$ with derivative in $\operatorname{Lip}(\eta), \eta>\frac{1}{2}$;

and in addition, with $\|\cdot\|$ denoting the Euclidean norm:

(iii) the $\varepsilon_{t}$ are independent and identically distributed vectors with mean zero, positive definite covariance matrix $\Omega$, and $E\left\|\varepsilon_{t}\right\|^{q}<\infty, q \geq 4, q>$ $2 /(2 \delta-1)$. 
This is precisely Assumption 1 of $\mathrm{RH}$, where it is discussed; it is a fairly general short memory specification for $u_{t}$, allowing weak convergence of fractional transforms, or central limit theorems, as required. In connection with this, denote by $W(r)$ the $2 \times 1$ vector Brownian motion with covariance matrix $\Omega$, and define, for $d>0$, (Type II-see Marinucci and Robinson, 1999b) fractional Brownian motion

$$
W(r ; d)=\int_{0}^{r} \frac{(r-s)^{d-1}}{\Gamma(d)} d W(s),
$$

and then define $\bar{W}(r ; d)$ and $\widetilde{W}(r ; d)$ to respectively be the first and second elements of the vector $A(1) W(r ; d)$.

Define

$$
\begin{aligned}
\Phi_{1} & =\int_{0}^{1} \widetilde{W}(r ; \delta)^{2} d r, \\
\Phi_{2} & =\mu_{2 \ddagger} \int_{0}^{1} r^{\phi_{2 \ddagger}-1 / 2} \widetilde{W}(r ; \delta) d r, \\
\Phi_{3} & =\mu_{2 \ddagger}^{2}\left(2 \phi_{2 \ddagger}\right)^{-1},
\end{aligned}
$$

and

$$
\begin{aligned}
& \Psi_{1}=\int_{-\pi}^{\pi}\left(1-e^{i \lambda}\right)^{-\gamma}\left(1-e^{-i \lambda}\right)^{-\delta} f_{12}(\lambda) d \lambda, \\
& \Psi_{2}=f_{12}(0) \sin \delta \pi,
\end{aligned}
$$

where $f_{12}(\lambda)$ is the $(1,2)$ th element of $f(\lambda), \Psi_{3}$ is such that for $\gamma>1 / 2$

$$
\Psi_{3}=\int_{0}^{1} \bar{W}(r ; \gamma) \widetilde{W}(r ; \delta) d r,
$$

and for $\gamma \leq 1 / 2 \Psi_{3}$ is an $O_{p}(1)$ random variable and

$$
\begin{aligned}
& \Psi_{4}=\left\{\begin{array}{cl}
\mu_{2 \ddagger} \int_{0}^{1} r^{\phi_{2 \ddagger}-1 / 2} \bar{W}(r ; \gamma) d r & \text { if } \gamma>0, \\
\mu_{2 \ddagger} \int_{0}^{1} r^{\phi_{2 \ddagger}-1 / 2} d \bar{W}(r) & \text { if } \gamma=0,
\end{array}\right. \\
& \Psi_{5}=\mu_{1 \dagger} \int_{0}^{1} r^{\phi_{1 \dagger}-1 / 2} \widetilde{W}(r ; \delta) d r, \\
& \Psi_{6}=\frac{\mu_{1 \dagger} \mu_{2 \ddagger}}{\phi_{1 \dagger}+\phi_{2 \ddagger}} \text {. }
\end{aligned}
$$


Also define

$$
\begin{aligned}
& \Phi_{1}^{*}=\Phi_{1} 1\left(\delta \geq \phi_{2 \ddagger}\right), \\
& \Phi_{2}^{*}=\Phi_{2} 1\left(\delta=\phi_{2 \ddagger}\right), \\
& \Phi_{3}^{*}=\Phi_{3} 1\left(\delta \leq \phi_{2 \ddagger}\right),
\end{aligned}
$$

and

$$
\begin{aligned}
\Psi_{1}^{*}= & \Psi_{1} 1\left(\gamma+\delta<1, \gamma+\phi_{2 \ddagger} \leq 1, \phi_{1 \dagger}+\delta \leq 1, \phi_{1 \dagger}+\phi_{2 \ddagger} \leq 1\right), \\
\Psi_{2}^{*}= & \Psi_{2} 1\left(\gamma+\delta=1, \gamma \geq \phi_{1 \dagger}, \phi_{2 \ddagger} \leq \delta<1\right), \\
\Psi_{3}^{*}= & \Psi_{3} 1\left(\gamma+\delta>1, \gamma \geq \phi_{1 \dagger}, \delta \geq \phi_{2 \ddagger}\right), \\
\Psi_{4}^{*}= & \Psi_{4} 1\left(\gamma+\delta>1, \phi_{1 \dagger} \leq \gamma, \delta \leq \phi_{2 \ddagger}\right) \\
& +\Psi_{4} 1\left(\gamma+\delta=1 \text { and } \gamma>0, \phi_{1 \dagger} \leq \gamma, \delta<\phi_{2 \ddagger}\right) \\
& +\Psi_{4} 1\left(\gamma+\delta<1, \phi_{1 \dagger} \leq \gamma, 1 \leq \phi_{2 \ddagger}+\gamma\right), \\
\Psi_{5}^{*}= & \Psi_{5} 1\left(\gamma+\delta>1 \text { or } \gamma=0 \text { and } \delta=1, \phi_{1 \dagger} \geq \gamma, \phi_{2 \ddagger} \leq \delta\right) \\
& +\Psi_{5} 1\left(\gamma+\delta=1 \text { and } \gamma>0, \gamma<\phi_{1 \dagger}, \phi_{2 \ddagger} \leq \delta\right) \\
& +\Psi_{5} 1\left(\gamma+\delta<1, \phi_{2 \ddagger} \leq \delta, 1 \leq \phi_{1 \dagger}+\delta\right), \\
& \Psi_{6} 1\left(\gamma+\delta>1 \text { or } \gamma=0 \text { and } \delta=1, \phi_{1 \dagger} \geq \gamma, \phi_{2 \ddagger} \geq \delta\right) \\
& +\Psi_{6} 1\left(\gamma+\delta=1 \text { and } \gamma>0, \phi_{1 \dagger} \geq \gamma, \phi_{2 \ddagger} \geq \delta, \phi_{1 \dagger}+\phi_{2 \ddagger}>1\right) \\
& +\Psi_{6} 1\left(\gamma+\delta<1, \phi_{1 \dagger} \geq \gamma, \phi_{2 \ddagger} \geq \delta, \phi_{1 \dagger}+\phi_{2 \ddagger} \geq 1\right) .
\end{aligned}
$$

Introduce the sequences

$$
\begin{aligned}
k_{n} & =n^{\max \left(\delta, \phi_{2 \ddagger}\right)}, \\
\ell_{n} & =n^{\max \left(1, \gamma+\delta, \gamma+\phi_{2 \ddagger}, \delta+\phi_{1 \dagger}, \phi_{1 \dagger}+\phi_{2 \ddagger}\right)}+n \log n 1(\gamma+\delta=1, \gamma>0) .
\end{aligned}
$$

Theorem 1. Let (8), (9), (12), (13) and Assumption 1 hold. Then as $n \rightarrow \infty$,

$$
\left(k_{n}^{2} / \ell_{n}\right)(\bar{\nu}-\nu) \Rightarrow\left\{\Psi_{1}^{*}+\Psi_{2}^{*}+\Psi_{3}^{*}+\Psi_{4}^{*}+\Psi_{5}^{*}+\Psi_{6}^{*}\right\} /\left\{\Phi_{1}^{*}+2 \Phi_{2}^{*}+\Phi_{3}^{*}\right\},
$$

where by " $\Rightarrow$ " we mean convergence in the Skorohod $J_{1}$ topology.

We can deduce from Theorem 1 exact rates of convergence, if any, of $\bar{\nu}$ to $\nu$. Let $O_{e}($.$) denote exact rate of convergence in probability. We have:$

$$
\begin{aligned}
& \bar{\nu}=\nu+O_{e}\left(n^{1-2 \delta}\right), \quad \text { if } \delta \geq \phi_{2 \ddagger}, \gamma+\delta<1 \text { and } \delta+\phi_{1 \dagger} \leq 1 \\
& \bar{\nu}=\nu+O_{e}\left(n^{1-2 \delta} \log n\right), \quad \text { if } \delta \geq \phi_{2 \ddagger}, \gamma+\delta=1 \text { and } \gamma \geq \phi_{1 \dagger} \\
& \bar{\nu}=\nu+O_{e}\left(n^{\gamma-\delta}\right), \quad \text { if } \delta \geq \phi_{2 \ddagger}, \gamma \geq \phi_{1 \dagger} \text { and } \gamma+\delta>1 \\
& \bar{\nu}=\nu+O_{e}\left(n^{\phi_{1 \dagger}-\delta}\right), \quad \text { if } \delta \geq \phi_{2 \ddagger}, \phi_{1 \dagger} \geq \gamma \text { and } \gamma+\delta>1 \\
& \quad \text { or } \delta \geq \phi_{2 \ddagger}, \phi_{1 \dagger}+\delta \geq 1 \text { and } \gamma+\delta<1 \\
& \bar{\nu}=\nu+O_{e}\left(n^{1-2 \phi_{2 \ddagger}}\right), \quad \text { if } \phi_{2 \ddagger} \geq \delta, \gamma+\phi_{2 \ddagger}<1 \text { and } \phi_{1 \dagger}+\phi_{2 \ddagger} \leq 1 \\
& \bar{\nu}=\nu+O_{e}\left(n^{\gamma-\phi_{2 \ddagger}}\right), \quad \text { if } \phi_{2 \ddagger} \geq \delta, \gamma \geq \phi_{1 \dagger} \text { and } \gamma+\phi_{2 \ddagger}>1 \\
& \bar{\nu}=\nu+O_{e}\left(n^{\phi_{1 \dagger}-\phi_{2 \ddagger}}\right), \quad \text { if } \phi_{2 \ddagger} \geq \delta, \phi_{1 \dagger} \geq \gamma \text { and } \phi_{1 \dagger}+\phi_{2 \ddagger}>1 .
\end{aligned}
$$


It follows that $\bar{\nu}$ is not even consistent when

$$
\phi_{1 \dagger} \geq \max \left(\delta, \phi_{2 \ddagger}\right),
$$

(see cases (55) and (58)) so that the deterministic trend in the cointegrating equation (8) dominates both the stochastic and deterministic trends in $x_{t}$. Otherwise, $\bar{\nu}$ is consistent. In case (55), with $\gamma<\phi_{1 \dagger}<\delta$, the deterministic trend in (8) worsens matters relative to the rates in RM, whereas in cases (52)-(54) the same rates are achieved, stochastic trends dominating. With $\phi_{2 \ddagger}>\delta$, the dominating deterministic trend in $x_{t}$ improves rates relative to RM, in (56) and (57), while in (58) both deterministic trends dominate and the rate simply reflects the extent to which $x_{t}$ 's trend dominates that in (8).

We can deduce from Theorem 1 more precise results. These are complicated in case of equalities $\phi_{2 \ddagger}=\delta$ and/or $\phi_{1 \dagger}=\gamma$, so we look only at strict inequalities. With $\gamma>\phi_{1 \dagger}, \delta>\phi_{2 \ddagger}$ the limit distributions corresponding to (52), (53) and (54) are identical to those of RM Propositions 6.1, 6.2 and 6.5. In case (57), with $\gamma>\phi_{1 \dagger}$ and $\phi_{2 \ddagger}>\delta$, stochastic trends dominate in (8) and deterministic ones in (9), and $n^{\phi_{2 \ddagger}-\gamma}(\bar{\nu}-\nu)$ converges to the normal variates $\Phi_{3}^{-1} \Psi_{4}$ (cf. Robinson and Marinucci, 2000) while in (58) with $\phi_{1 \dagger}>\gamma, \phi_{2 \ddagger}>\delta, n^{\phi_{2 \ddagger}-\phi_{1 \dagger}}(\bar{\nu}-\nu)$ converges to the constant $\Phi_{3}^{-1} \Psi_{6}$. The familiar case in which $x_{t}$ contains a unit root plus linear trend, and cointegrating errors that are $I(0)(\gamma=0, \delta=1$, $\left.\phi_{1 \dagger}=0.5, \phi_{2 \ddagger}=1.5\right)$ comes under (58).

\section{LEAST SQUARES ESTIMATES OF MODEL II}

The OLS estimate of $\nu_{+}=\left(\nu, \mu_{1}^{\prime}\right)^{\prime}$ in Model II (22) is

$$
\widetilde{\nu}_{+}=M_{x_{+} x_{+}}^{-1} M_{x_{+} y},
$$

where $x_{+t}=\left(x_{t}, g_{1}^{\prime}(t)\right)^{\prime}$. Define the sequence

$$
m_{n}=\left\{n^{\max \left(1, \gamma+\delta, \gamma+\phi_{2 \ddagger}\right)}+n \log n 1(\gamma+\delta=1, \gamma \neq 0)\right\},
$$

and the matrix sequences

$$
\begin{aligned}
B_{n} & =\left[\begin{array}{ll}
k_{n} & 0 \\
0 & D_{1 n}(0)
\end{array}\right], C_{n}=\left[\begin{array}{ll}
m_{n} / k_{n} & 0 \\
0 & n^{\gamma} I_{p_{11}}
\end{array}\right], \\
D_{i n}(d) & =\operatorname{diag}\left\{n^{\phi_{i 1}+d}, \ldots, n^{\phi_{i p_{i 1}}}+d\right\}, i=1,2 .
\end{aligned}
$$

For $i, j=1,2$, define the $p_{i 1} \times p_{j 1}$ matrix $\Xi_{i j}(c, d)$, having $(k, l)$ th element $\left(\phi_{i k}+\phi_{j l}-c-d\right)^{-1}$, and the $p_{11} \times 1$ vector $\Upsilon$, having $k$ th element $\left(\phi_{1 k}+\phi_{2 \ddagger}\right)^{-1}$. 
Also define

$$
\begin{gathered}
\Phi_{4}=\int_{0}^{1} g_{1}(r) \widetilde{W}(r ; \delta) d r, \\
\Phi_{5}=\mu_{2 \ddagger} \Upsilon, \\
\Phi_{6}=\Xi_{11}(0,0) ; \\
\Psi_{7}=\int_{0}^{1} \widetilde{W}(r ; 1) d \bar{W}(r)+\sum_{j=-\infty}^{0} \operatorname{Cov}\left(u_{10}, u_{2 j}\right), \quad \delta=1, \\
=\int_{0}^{1} \widetilde{W}(r ; \delta) d \bar{W}(r), \quad \delta>1 ; \\
\Psi_{8}=\left\{\begin{array}{l}
\int_{0}^{1} g_{1}(r) \bar{W}(r ; \gamma) d r \quad \text { if } \gamma>0 \\
\int_{0}^{1} g_{1}(r) d \bar{W}(r) \quad \text { if } \gamma=0 .
\end{array}\right.
\end{gathered}
$$

Let

$$
\begin{aligned}
& \Phi_{4}^{*}=\Phi_{4} 1\left(\delta \geq \phi_{2 \ddagger}\right), \\
& \Phi_{5}^{*}=\Phi_{5} 1\left(\delta \leq \phi_{2 \ddagger}\right),
\end{aligned}
$$

and

$$
\Phi=\left(\begin{array}{cc}
\Phi_{1}^{*}+\Phi_{2}^{*}+\Phi_{3}^{*} & \Phi_{4}^{* \prime}+\Phi_{5}^{* \prime} \\
\Phi_{4}^{*}+\Phi_{5}^{*} & \Phi_{6}
\end{array}\right)
$$

and also

$$
\begin{aligned}
\Psi_{1}^{* *}= & \Psi_{1} 1\left(\gamma+\delta<1, \gamma+\phi_{2 \ddagger} \leq 1\right), \\
\Psi_{2}^{* *}= & \Psi_{2} 1\left(\gamma+\delta=1, \phi_{2 \ddagger} \leq \delta<1\right), \\
\Psi_{3}^{* *}= & \Psi_{3} 1\left(\gamma+\delta>1, \delta \geq \phi_{2 \ddagger}, \gamma>0\right), \\
\Psi_{4}^{* *}= & \Psi_{4} 1\left(\gamma+\delta>1, \delta \leq \phi_{2 \ddagger}\right) \\
& +\Psi_{4} 1\left(\gamma+\delta=1 \text { and } \gamma>0, \delta<\phi_{2 \ddagger}\right) \\
& +\Psi_{4} 1\left(\gamma+\delta<1 \text { or } \gamma=0 \text { and } \delta=1, \phi_{2 \ddagger}+\gamma \geq 1\right), \\
\Psi_{7}^{* *}= & \Psi_{7} 1\left(\gamma=0, \delta \geq \max \left(\phi_{2 \ddagger}, 1\right)\right),
\end{aligned}
$$

and

$$
\Psi=\left(\begin{array}{c}
\Psi_{1}^{* *}+\Psi_{2}^{* *}+\Psi_{3}^{* *}+\Psi_{4}^{* *}+\Psi_{7}^{* *} \\
\Psi_{8}
\end{array}\right)
$$

To avoid multicollinearity in case $x_{t}$ is dominated by an element of $g_{2}(t)$ we introduce

Assumption 2. If $\delta<\phi_{2 \ddagger}$ then $\phi_{2 \ddagger} \neq \phi_{1 k}$ for $k=1, \ldots, p_{11}$. 
Theorem 2. Let (8), (9), (12), (13) and Assumptions 1 and 2 hold. Then as $n \rightarrow \infty$,

$$
C_{n}^{-1} \Phi B_{n}\left(\widetilde{\nu}_{+}-\nu_{+}\right) \Rightarrow \Psi .
$$

The cumbersome norming (where indeed $\Phi$ can be stochastic) is to enable a neat, general statement, $C_{n}$ and $B_{n}$ not commuting with the non-diagonal matrix $\Phi$, but we can readily deduce more comprehensible conclusions. Due to the sufficient accounting for deterministic trends in (8), $\widetilde{\nu}$ is always consistent. We have the following cases, that we classify according to whether the stochastic or deterministic component of $x_{t}$ dominates:

$$
\begin{aligned}
& S: \delta \geq \phi_{2 \ddagger} \\
& D: \delta \leq \phi_{2 \ddagger}
\end{aligned}
$$

$$
\begin{aligned}
S: & \\
& \widetilde{\nu}-\nu=O_{e}\left(n^{1-2 \delta}\right), \quad \text { if } \delta \geq \phi_{2 \ddagger}, \gamma+\delta<1, \\
& \widetilde{\nu}-\nu=O_{e}\left(n^{1-2 \delta} \log n\right), \quad \text { if } \delta \geq \phi_{2 \ddagger}, \gamma+\delta=1, \gamma>0, \\
& \widetilde{\nu}-\nu=O_{e}\left(n^{-1}\right), \quad \text { if } \gamma=1-\delta=0, \phi_{2 \ddagger} \leq 1, \\
& \widetilde{\nu}-\nu=O_{e}\left(n^{\gamma-\delta}\right), \quad \text { if } \delta \geq \phi_{2 \ddagger}, \gamma+\delta>1, \\
D: & \\
& \widetilde{\nu}-\nu=O_{e}\left(n^{1-2 \phi_{2 \ddagger}}\right), \quad \text { if } \delta<\phi_{2 \ddagger}, \gamma+\phi_{2 \ddagger} \leq 1, \\
& \widetilde{\nu}-\nu=O_{e}\left(n^{-1}\right), \quad \text { if } \delta<\phi_{2 \ddagger}=1, \gamma=0, \\
& \widetilde{\nu}-\nu=O_{e}\left(n^{\gamma-\phi_{2 \ddagger}}\right), \quad \text { if } \delta \leq \phi_{2 \ddagger}, \gamma+\phi_{2 \ddagger}>1 .
\end{aligned}
$$

When $\delta>\phi_{2 \ddagger}$ the limit distributions corresponding to (79)-(82) are those of RM (see Propositions 6.1, 6.2, 6.3, 6.4 and 6.5). When $\phi_{2 \ddagger}>\max (\delta, 1-\gamma)$ we deduce that

$$
\left[\begin{array}{cc}
n^{\phi_{2 \ddagger}-\gamma} & 0 \\
0 & D_{1 n}(-\gamma)
\end{array}\right]\left(\widetilde{\nu}_{+}-\nu_{+}\right) \rightarrow_{d}\left[\begin{array}{cc}
\Phi_{3} & \Phi_{5}^{\prime} \\
\Phi_{5} & \Phi_{6}
\end{array}\right]^{-1}\left[\begin{array}{l}
\Psi_{4} \\
\Psi_{8}
\end{array}\right],
$$

where the right side is a multivariate normal vector. The familiar case $\gamma=0$, $\delta=1, \phi_{1 \dagger}=0.5, \phi_{2 \ddagger}=1.5$ comes under (85).

\section{GENERALIZED LEAST SQUARES ESTIMATES OF MODEL III}

The estimates of Sections 2 and 3 are computationally convenient, especially as they both avoid the necessity of knowledge of memory parameters and do not require their estimation. However, even when they converge, their rates 
are not in general optimal, and their limit distributions are for the most part inconvenient for practical use.

To remedy these drawbacks we consider GLS estimation. For $c, d \geq 0$, define

$$
\begin{gathered}
z_{t}(c, d)=\left(y_{t}(c), x_{t}(d)\right)^{\prime} \\
w_{t}(c, d)=\left(\begin{array}{ccc}
\Delta^{c} x_{t} & \Delta^{c} g_{1}^{\prime}(t) & 0 \\
0 & 0 & \Delta^{d} g_{2}^{\prime}(t)
\end{array}\right)^{\prime}, \\
\nu_{++}=\left(\nu, \mu_{1}^{\prime}, \mu_{2}^{\prime}\right)^{\prime} .
\end{gathered}
$$

Thus (23), (24) can be written

$$
z_{t}(\gamma, \delta)=w_{t}^{\prime}(\gamma, \delta) \nu_{++}+v_{t},
$$

where now $v_{t}=\left(v_{1 t}(\gamma), v_{2 t}(\delta)\right)^{\prime}$. In RH the right hand side was simplified by the correct assumption that $\mu_{1}=0, \mu_{2}=0$ and $v_{t}=\left(u_{1 t}, u_{2 t}\right)^{\prime}$. Here we simultaneously estimate $\nu$ with $\mu_{1}, \mu_{2}$ and show that $s_{1 t}, s_{2 t}$ (defined after (25) in Section 1) exert negligible effect. As in $\mathrm{RH}$ we construct two kinds of estimate of GLS type that allow for flexible parametric modelling of $f(\lambda)$, that is, the autocorrelation structure of $u_{t}$, and that either depend on known $\gamma, \delta$ or allow substitution of estimates of these without affecting limiting distributional properties. One kind of estimate is 'time-domain', the other is 'frequency-domain', and the practitioner's choice between them is based on computational considerations and taste.

The time-domain estimate involves autoregressive (AR) transformation. From Assumption $1 u_{t}$ has an AR representation

$$
B(L) u_{t}=\varepsilon_{t},
$$

with $B(s)=I_{2}-\sum_{j=1}^{\infty} B_{j} s^{j}$, such that the $B_{j}$ are unknown $2 \times 2$ matrices. We know functions $\Omega(h), B_{j}(h)$, where $h \in \mathbb{R}^{p}, p \geq 1$, such that $\Omega=\Omega(\theta)$, $B_{j} \equiv B_{j}(\theta)$ for some $\theta \in \mathbb{R}$. Define $B(s ; h)=I_{2}-\sum_{j=1}^{\infty} B_{j}(h) s^{j}$ and then

$$
\begin{aligned}
\widetilde{a}(c, d, h) & =\sum_{t}\left\{B(L ; h) w_{t}^{\prime}(c, d)\right\}^{\prime} \Omega(h)^{-1}\left\{B(L ; h) z_{t}(c, d)\right\}, \\
\widetilde{b}(c, d, h) & =\sum_{t}\left\{B(L ; h) w_{t}^{\prime}(c, d)\right\}^{\prime} \Omega(h)^{-1}\left\{B(L ; h) w_{t}^{\prime}(c, d)\right\} .
\end{aligned}
$$

Each of the AR transformations is truncated since $w_{t}(c, d)=0, z_{t}(c, d)=0$, $t \leq 0$. Now write

$$
\widetilde{\nu}_{++}(c, d, h)=\widetilde{b}(c, d, h)^{-1} \widetilde{a}(c, d, h)
$$

and consider

$$
\widetilde{\nu}_{++}(\gamma, \delta, \theta), \widetilde{\nu}_{++}(\gamma, \delta, \widehat{\theta}), \widetilde{\nu}_{++}(\widehat{\gamma}, \delta, \widehat{\theta}), \widetilde{\nu}_{++}(\gamma, \widehat{\delta}, \widehat{\theta}), \widetilde{\nu}_{++}(\widehat{\gamma}, \widehat{\delta}, \widehat{\theta}),
$$

for given estimates $\widehat{\gamma}, \widehat{\delta}, \widehat{\theta}$. These estimates of $\nu_{++}$respectively cover the cases in which $\gamma, \delta$ and $\theta$ are all known, $\gamma$ and $\delta$ are known but $\theta$ is not, only $\delta$ 
is known, only $\gamma$ is known, and $\gamma, \delta$ and $\theta$ is all unknown. Thus $\widetilde{\nu}_{++}(\gamma, \delta, \widehat{\theta})$ covers the familiar case where $\gamma=0, \delta=1$ is known, and $u_{t}$ is, say, white noise or $\operatorname{AR}(1) ; \widetilde{\nu}_{++}(\widehat{\gamma}, \delta, \widehat{\theta})$ with $\delta=1$ accepts the evidence of unit root behaviour suggested by a number of empirical studies of macroeconomic variables but allows for the possibility of long memory or mean-reversion in the cointegrating error; $\widetilde{\nu}_{++}(0, \widehat{\delta}, \widehat{\theta})$ insists only that the cointegrating error has short memory, possibly white noise; and $\widetilde{\nu}_{++}(\widehat{\gamma}, \widehat{\delta}, \widehat{\theta})$ is completely agnostic.

When $u_{t}$ is a not a finite-degree AR process $\widetilde{\nu}_{++}$can still be computed, but the following frequency-domain estimates may be preferable, making use of the neat form of the spectral density matrix $f(\lambda)$ when $u_{t}$ is a finite-degree moving average (MA) or autoregressive moving average (ARMA) sequence, or in some other models. Let $f(\lambda ; h)$ be a known function of $\lambda \in(-\pi, \pi]$ and $h \in \mathbb{R}^{p}$, such that $f(\lambda ; \theta)=f(\lambda)$, so

$$
f(\lambda ; h)=(2 \pi)^{-1} B\left(e^{i \lambda} ; h\right)^{-1} \Omega(h) B\left(e^{-i \lambda} ; h\right)^{-1^{\prime}} .
$$

Define the discrete Fourier transforms

$$
F_{w(c, d)}(\lambda)=\frac{1}{(2 \pi n)^{\frac{1}{2}}} \sum_{t} w_{t}(c, d) e^{i t \lambda}, \quad F_{z(c, d)}(\lambda)=\frac{1}{(2 \pi n)^{\frac{1}{2}}} \sum_{t} z_{t}(c, d) e^{i t \lambda} .
$$

Put

$$
\begin{aligned}
a(c, d, h) & =\sum_{j} F_{w(c, d)}\left(\lambda_{j}\right) f\left(\lambda_{j} ; h\right)^{-1} F_{z(c, d)}\left(-\lambda_{j}\right), \\
b(c, d, h) & =\sum_{j} F_{w(c, d)}\left(\lambda_{j}\right) f\left(\lambda_{j} ; h\right)^{-1} F_{w(c, d)}^{\prime}\left(-\lambda_{j}\right) .
\end{aligned}
$$

Define

$$
\widehat{\nu}_{++}(c, d, h)=b(c, d, h)^{-1} a(c, d, h) .
$$

Consider the frequency-domain variants of the five estimates (95),

$$
\widehat{\nu}_{++}(\gamma, \delta, \theta), \widehat{\nu}_{++}(\gamma, \delta, \widehat{\theta}), \widehat{\nu}_{++}(\widehat{\gamma}, \delta, \widehat{\theta}), \widehat{\nu}_{++}(\gamma, \widehat{\delta}, \widehat{\theta}), \widehat{\nu}_{++}(\widehat{\gamma}, \widehat{\delta}, \widehat{\theta}) .
$$

When $v_{t}=\left(v_{1 t}, v_{2 t}\right)^{\prime}$ is a priori white noise, $\widetilde{\nu}(c, d, h) \equiv \widehat{\nu}(c, d, h)$.

To handle the last four estimates in (95) and (101) we introduce the following further assumptions. Denote by $\Theta$ the compact set of all admissible values of $\widehat{\theta}$.

\section{Assumption 3.}

(i) $f(\lambda ; \theta)=f(\lambda)$.

(ii) $f(\lambda ; h)$ has determinant bounded away from zero on $([-\pi, \pi] \times \Theta)$.

(iii) $f(\lambda ; h)$ is boundedly differentiable in $h$ on $([-\pi, \pi] \times \Theta)$, with derivative that is continuous in $h$ at $h=\theta$ for all $\lambda$. 
(iv) $f(\lambda ; \theta)$ is differentiable in $\lambda$, with derivative satisfying a Lipschitz condition of order greater than $1 / 2$ in $\lambda$.

(v) $(\partial / \partial h) f(\lambda ; h)$ is differentiable in $\lambda$ at $h=\theta$, with derivative satisfying a Lipschitz condition of order greater than $1 / 2$ in $\lambda$.

\section{Assumption 4.}

(i) There exists $K<\infty$ such that

$$
|\widehat{\gamma}|+|\widehat{\delta}| \leq K
$$

and $\rho>\max (0,1-\beta)$ such that

$$
\widehat{\gamma}=\gamma+O_{p}\left(n^{-\rho}\right), \widehat{\delta}=\delta+O_{p}\left(n^{-\rho}\right) .
$$

(ii)

$$
\widehat{\theta}=\theta+O_{p}\left(n^{-\frac{1}{2}}\right), \text { where } \theta \in \Theta .
$$

These are identical to assumptions in $\mathrm{RH}$, where they are discussed. However in view of the presence of deterministic trends in our model we need to say more about Assumption 4. In general it seems that if we carry out the procedure of Velasco and Robinson (2000) to estimate $\delta$, but based on residuals from the OLS regression of $x_{t}$ on $g_{2}(t)$, we will achieve the second part of (103) with $\rho=\frac{1}{2}$. As is often the case with justifying insertion of residuals in implicitly-defined estimation procedures, the proof is rather tedious so the details are not pursued. Alternatively, if all $\phi_{2 j}-\frac{1}{2}$ in $g_{2}(t)$ are integers, the use of Velasco and Robinson's (2000) procedure based on the raw $x_{t}$, but using a Kolmogorov taper of sufficiently high order, will exactly remove such a polynomial trend. Strictly, this does not require knowledge of $\phi_{21}$, but rather of the largest value we might anticipate for $\phi_{21}$. On the other hand, we might carry out the initial OLS estimation by including such arbitrarily large powers of $t$, so there may not be a great deal to choose between the two approaches. The higher the taper order, the greater the imprecision in estimation of $\delta$, while inclusion of unnecessary regressors in the OLS approach is liable to have similar effect. The OLS approach has the advantage over tapering that non-integral powers of $t$ may be employed, while tapering seems to be needed anyway to estimate large enough values of $\delta$ in the Velasco and Robinson (2000) approach. So far as the first part of (103) is concerned, Velasco and Robinson's (2000) approach can again be modified to estimate $\gamma$, with the use of residuals from the regression of $y_{t}$ on $x_{t}$ and $g_{2}(t)$ apparently necessary, while tapering is then unnecessary if $\gamma<\frac{1}{2}$ is anticipated. Recently, Chen and Hurvich (2003) have employed tapers in estimating the cointegrating coefficient in a fractional system 
with deterministic trends. However, they are concerned with estimating the relation between underlying (possibly nonstationary) stochastic components that are corrupted by deterministic trends. These (assumed to be polynomial in t) are handled by differencing, with tapering then employed in a modification of the narrow-band least squares regression estimate of Robinson (1994). Earlier, Robinson (1986) had employed tapering in reducing errors-in-variables bias in band-spectrum regression in which (contrary to Robinson (1994)) bands do not degenerate asymptotically and the processes have short memory.

Define

$$
\begin{aligned}
& \Lambda_{i}(d)=\operatorname{diag}\left\{\frac{\Gamma\left(\phi_{i 1}+1 / 2\right)}{\Gamma\left(\phi_{i 1}-d+1 / 2\right)}, \ldots, \frac{\Gamma\left(\phi_{i p_{i 1}}+1 / 2\right)}{\Gamma\left(\phi_{i p_{i 1}}-d+1 / 2\right)}\right\}, \quad i=1,2, \\
& Q(r)=\left(\begin{array}{cc}
\widetilde{W}(r ; \beta) 1\left(\delta \geq \phi_{2 \ddagger}\right)+\mu_{2 \ddagger} \frac{\Gamma\left(\phi_{2 \ddagger}+1 / 2\right)}{\Gamma\left(\phi_{2 \ddagger}-\gamma+1 / 2\right)} r^{\phi_{2 \ddagger}-\gamma-1 / 2} 1\left(\delta \leq \phi_{2 \ddagger}\right) & 0 \\
\Lambda_{1}(\gamma) g_{1}(r) r^{-\gamma} & 0 \\
0 & \Lambda_{2}(\delta) g_{2}(r) r^{-\delta}
\end{array}\right.
\end{aligned}
$$

and introduce the matrix sequence

$$
D_{n}=\left(\begin{array}{ccc}
n^{\max \left(\delta, \phi_{2 \ddagger}\right)-\gamma} & 0 & 0 \\
0 & D_{1 n}(-\gamma) & 0 \\
0 & 0 & D_{2 n}(-\delta)
\end{array}\right) .
$$

Theorem 3. Let (8), (9), (12), (25) and Assumptions 1-4 hold, with $q>$ $1 /(2 \beta-1)$ in Assumption 1. Then, denoting by $\widehat{\nu}_{++}^{*}$ any of the estimates in (95) or (101), we have as $n \rightarrow \infty$,

$$
D_{n}\left(\widehat{\nu}_{++}^{*}-\nu_{++}\right) \Rightarrow\left\{\int_{0}^{1} Q(r) f(0)^{-1} Q^{\prime}(r) d r\right\}^{-1} 2 \pi \int_{0}^{1} Q(r) B(1)^{\prime} \Omega^{-1} d W(r) .
$$

As in $\mathrm{RH}$ we find that we can estimate $\nu$, along with $\mu_{1}, \mu_{2}$, as well without knowing $\gamma$ and/or $\delta$ and/or $\theta$ as knowing them, so that efficiency of estimation of $\gamma, \delta$ and $\theta$ does not matter. When $\delta>\phi_{2 \ddagger}$, we have precisely the same limit distribution and rate of convergence for $\hat{\nu}^{*}$ as the estimate of $\mathrm{RH}$, which ignores the possibility of trends, and the same limit distribution. The distribution is changed when $\delta=\phi_{2 \ddagger}$, but the rate of convergence is not. When $\delta<\phi_{2 \ddagger}$ the rate is faster than in $\mathrm{RH}$. When $\delta>\phi_{2 \ddagger}$ there is mixed normal asymptotics as in $\mathrm{RH}$, as there is also for $\delta=\phi_{2 \ddagger}$, while when $\delta<\phi_{2 \ddagger}$ the limit distribution is normal, as in the familiar case $\gamma=0, \delta=1, \phi_{1 \dagger}=0.5, \phi_{2 \ddagger}=1.5$. Thus in all cases we can expect Wald tests on $\nu_{++}$, for example tests on $\nu$ (such as $\nu=1$ as in PPP testing) or on $\mu_{1}$ (e.g. $\mu_{1}=0$, to test whether deterministic trends affect $y_{t}$ only through $x_{t}$ ), to have standard, $\chi^{2}$, asymptotics. Our theory differs from that of Hansen (1992) (who assumed known $\gamma=0, \delta=1$ integer powers of 
$t$ ) in that he allowed $x_{t}$ to be a vector and assumed its dimension greater than the number of deterministic regressors. This is impossible in our set-up. As in $\mathrm{RH}$ we do not allow $x_{t}$ to be a vector because an interesting treatment of this case would entail, in our fractional setting, allowance for differing integration orders in the elements of $x_{t}$, and also two or more cointegrating relationships, possibly with different integration orders.

In general introduce the null hypothesis:

$$
H_{0}: E \nu_{++}=e,
$$

where $E$ is a given $q \times\left(1+p_{11}+p_{21}\right)$ matrix of rank $q<1+p_{11}+p_{21}$ and $e$ is a given $q \times 1$ vector.

Corollary 4. Denoting by $b^{*}$ any of the quantities $\widetilde{b}(c, d, h), b(c, d, h)$, with $c=\gamma$ or $\widehat{\gamma}, d=\delta$ or $\widehat{\delta}$ and $h=\theta$ or $\widehat{\theta}$, under (109) the Wald statistics

$$
\left(E \widehat{\nu}_{++}^{*}-e\right)^{\prime}\left(E b^{*-1} E^{\prime}\right)^{-1}\left(E \widehat{\nu}_{++}^{*}-e\right) \rightarrow_{d} \chi_{q}^{2}, \text { as } n \rightarrow \infty .
$$

\section{MONTE CARLO SIMULATIONS}

We have encountered convergence rates and limit distributions that can vary substantially across both types of estimate and memory characteristics of the data generating process. In order to investigate how reliable a guide the asymptotic theory is in moderate-sized samples a small Monte Carlo study was carried out. We generated data from (8) and (9) for several combinations of stochastic and deterministic trends. Two different specifications for each equation were employed. For the cointegrating equation (8) these were:

DT1a: $\quad p_{1}=1 ; \quad \mu_{11}=1 ; \quad \phi_{11}=0.5$.

DT1b: $\quad p_{1}=1 ; \quad \mu_{11}=\mu_{12}=1 ; \quad \phi_{12}=0.5, \phi_{11}=1.5$.

For the $x$ equation (9) they were

$$
\begin{array}{ll}
D T 2 a: & p_{2}=1 ; \quad \mu_{21}=1 ; \quad \phi_{21}=1.5 . \\
D T 2 b: & p_{2}=2 ; \quad \mu_{21}=\mu_{22}=1 ; \quad \phi_{22}=1.5, \phi_{21}=2.5 .
\end{array}
$$

Thus DT1a consists only of an intercept, while $D T 1 b$ is a time trend; $D T 2 a$ and $D T 2 b$ include no constants, the former consisting only of a linear term, the latter a linear and a quadratic. We employed all four combinations, $D T a=$ $D T 1 a \times D T 2 a, D T b=D T 1 a \times D T 2 b, D T c=D T 1 b \times D T 2 a$ and $D T d=$ $D T 1 b \times D T 2 b$. The stochastic component of the model was specified as follows. 
We took $A_{j} \equiv 0, j \geq 1$, in Assumption 1 where the covariance matrix $\Omega$ of Gaussian $u_{t}=\varepsilon_{t}$ was given by

$$
\Omega=\left[\begin{array}{cc}
\tau & \rho \tau^{\frac{1}{2}} \\
\rho \tau^{\frac{1}{2}} & 1
\end{array}\right]
$$

for varying $\rho, \tau$. Our stochastic trends for the cointegrating equation and the $x$ equation were then determined by the following six choices of $(\gamma, \delta)$ :

$\begin{array}{llrl}\text { STa: } & & (\gamma, \delta)=(0,0.6) \\ \text { STb: } & (\gamma, \delta)=(0,1.2) \\ \text { STc: } & (\gamma, \delta)=(0,2) \\ \text { STd: } & (\gamma, \delta)=(0.4,0.6) \\ \text { STe: } & (\gamma, \delta)=(0.4,1.2) \\ \text { STf: } & (\gamma, \delta)=(0.4,2)\end{array}$

Note that $S T d$ is not covered by the theory for our GLS estimate. We considered each of the 24 combinations of the $4 D T$ 's and $6 S T$ 's. Finally we took $\nu=1$.

Tables 1-3 indicate rates of convergence of the various estimates of $\nu: \bar{\nu}$, the OLS estimate in Section 2; the first element $\widetilde{\nu}$ of the OLS estimate $\widetilde{\nu}_{+}$in Section 3 ; the first element $\widehat{\nu}$ of the generic GLS estimate $\widehat{\nu}_{++}^{*}$ in Section 4 (note indeed that in our case of white noise AR, (95) and (101) are identical). For comparison we include also the rates when there are no deterministic trends in either (8) or (9). Our design includes cases where such trends improve, leave unchanged, or reduce the rate for $\bar{\nu}$, or even make it inconsistent, and cases where rates for $\widetilde{\nu}$ and $\widehat{\nu}$ are either unchanged or improved. Nevertheless it would be possible to choose combinations that might seem more "interesting" in view of the various outcomes reported in Sections 2-4. Our choice is motivated by two factors. One is to enable comparison with the design of $\mathrm{RH}$, who used precisely the same $S T$ 's, and values of $\rho$ and $\tau$, with no DT's, and computed $\bar{\nu}$ and the GLS estimate of Section 4 simplified by (correctly) assuming no deterministic trends. The other is that non-fractional powers of $t$ in the $D T$ 's seem rather typical of current macroeconometric practice. However our simulations fall very far short indeed of providing a comprehensive study, especially as behaviour will vary with the $\mu_{i j}$ as well as the $\phi_{i j}$, not to mention being affected by the presence of short memory autocorrelation.

We computed the OLS estimates $\bar{\nu}$ and $\widetilde{\nu}$ described above, as well as infeasible and feasible GLS estimates of $\nu$, namely $\widehat{\nu}_{I}=\widehat{\nu}(\gamma, \delta, \widehat{\theta})$ and $\widehat{\nu}_{F}=\widehat{\nu}(\widehat{\gamma}, \widehat{\delta}, \widehat{\theta})$ where $\widehat{\nu}(c, d, h)$ is the first element of $\widehat{\nu}_{++}(c, d, h)$ with $\widehat{\gamma}, \widehat{\delta}$ and $\widehat{\theta}$ as follows.

Having obtained $\widetilde{\nu}_{+}=\left(\widetilde{\nu}, \widetilde{\mu}_{2}^{\prime}\right)^{\prime}$ we computed the $\widetilde{v}_{1 t}=y_{t}-\widetilde{\nu}_{+}^{\prime} x_{+t}$ and $\widetilde{v}_{2 t}=$ $x_{t}-\widetilde{\mu}_{2}^{\prime} g_{2}(t)$. For given $(c, d)$ define $\widetilde{u}_{t}(c, d)=\left(\widetilde{v}_{1 t}(c), \widetilde{v}_{2 t}(d)\right)^{\prime}$. Since $u_{t}$ is white noise, $\theta$ parameterizes only $\Omega$, for which we employ the estimates $\widetilde{\Omega}=\Omega_{(\gamma, \delta)}$ and $\widehat{\Omega}=\Omega_{(\widehat{\gamma}, \widehat{\delta})}$, where $\Omega_{(c, d)}=n^{-1} \sum_{t} \widetilde{u}_{t}(c, d) \widetilde{u}_{t}^{\prime}(c, d)$, the former referring to the case $(\gamma, \delta)$ known (i.e. $\left.\widehat{\nu}_{I}\right)$, the latter to the case $(\gamma, \delta)$ is estimated by $(\widehat{\gamma}, \widehat{\delta})$ (i.e. $\left.\widehat{\nu}_{F}\right)$. Here $\widehat{\gamma}$ was the Whittle pseudo-maximum likelihood estimate of Velasco and Robinson (2000) applied to the series $\widetilde{v}_{1 t}$ (without tapering the 
data). Likewise $\widehat{\delta}$ was obtained by the same method, but applied to the first difference of $\widetilde{v}_{2 t}$, then adding back 1 when $\delta=1.2$ or $\delta=2$ (as an alternative to tapering, again as in the simulations of $\mathrm{RH}$ ).

For the null hypothesis (109) we took $\nu=1$, and in the Wald statistic (110) we computed $W_{I}$ based on $\widehat{\nu}_{I}$ and $b(\gamma, \delta, \widetilde{\theta})$ and $W_{F}$ based on $\widehat{\nu}_{F}$ and $b(\widehat{\gamma}, \widehat{\delta}, \widehat{\theta})$, $\widetilde{\theta}$ and $\widehat{\theta}$ respectively denoting the vectors consisting of the three distinct elements of $\widetilde{\Omega}$ and $\widehat{\Omega}$. We employed sample sizes $n=64,125$ and 256, with 1000 replications.

We present two groups of simulations: in the first we keep $\tau=1$ and $\rho=0.5$ fixed, and consider the 24 combinations of deterministic and stochastic trends, while in the second group we focus on $\delta=0.6, \gamma=0$ and study the effect of alternative combinations of $\tau$ and $\rho$.

In Tables 4 and 5 we present Monte Carlo bias (the difference between the average of the estimates and $\nu$ ), and sample standard deviation for the first group of simulations. The stochastic component dominates in $x_{t}$ in 4 of the 24 combinations considered, namely when $\delta=2$ and $g_{2}(t)=t$ (for any $\gamma, g_{1}(t)$ ). In these situations the estimates have the same rate of convergence as in RH but the presence of $g_{1}(t)$ may affect the standard deviations of $\widetilde{\nu}, \widehat{\nu}_{I}$ and $\widehat{\nu}_{F}$. This is apparently a minor issue when the other regressors have a very low order, as it is for DTa $\left(g_{1}(t)=1\right)$, where the bias, standard deviation and empirical sizes of $\widetilde{\nu}, \widehat{\nu}_{I}$ and $\widehat{\nu}_{F}$ resemble those in $\mathrm{RH}$, but under $D T c$, which has a larger order for $g_{1}(t)$, we observed larger dispersion.

In the remaining 20 situations the estimates are dominated by the deterministic component in $x_{t}$, and consistency under DTc fails for $\bar{\nu}$ while $\widetilde{\nu}$ and GLS are not covered by the theory presented here. For the other three deterministic components, the faster rates of convergence result in much lower bias and standard deviation compared to the ones in RH. Contrary to the results in $\mathrm{RH}$, moving from $\delta=0.6$ to $\delta=1.2$ (and to $\delta=2$ too, when $D T b$ and $D T d$, i.e. $g_{2}(t)=t+t^{2}$, are considered) does not greatly affect precision. Increasing $\gamma$ from 0 to 0.4 has a visible, if rather small, effect, and again this is stable regardless of $\delta$, so long as the deterministic component dominates in $x_{t}$.

In general the OLS estimates are subject to bias due to correlation between $u_{1 t}$ and $u_{2 t}$, and in $\bar{\nu}$ there is also the additional component due to omission of $g_{1}(t)$ : both should induce a positive bias given the values of $\mu_{1}$ and of $\rho$, but we find that this is small. The bias of $\widetilde{\nu}$ is even smaller. Finally, we find that $\widehat{\nu}_{I}$ is slightly more precise than $\widehat{\nu}_{F}$, and that both are superior to $\widetilde{\nu}$, but in all cases considered the standard deviation in within the same range.

In Table 6 we present empirical sizes, for nominal sizes $\alpha=0.05$ and $\alpha=0.10$, of $\widehat{\nu}_{I}$ and $\widehat{\nu}_{F}$. As in $\mathrm{RH}$, those for $\widehat{\nu}_{I}$ are fairly precise, but those for $\widetilde{\nu}_{F}$ tend to be too big, though the difference gets smaller the larger the sample is. The difference in rates of convergence does not seem to affect the pattern, which is rather stable among all combinations. It is interesting to observe that under $D T a, D T b$ and $D T d$, the case $\delta=0.6, \gamma=0.4$ does not behave differently from the others, contrary to the simulations in RH. 
In the second part of the simulation exercise, we consider the effects of alternative specifications of the matrix $\Omega$. Notice that we have $E\left(u_{2 t}\right)=1$ and so altering $\tau$ affects the dispersion of $u_{1 t}$, inverting the design of $\mathrm{RH}$; due to the dominating deterministic component, changing the variance of $u_{2 t}$ would not affect the results in a relevant way. In fact, we already remarked that even increasing $\delta$ from 0.6 to 1.2 (and to 2 under $D T d$ ) for given $\gamma$ has no major effect on variability, and this will be much more the case if $\delta$ is unchanged.

We present bias in Table 7 , standard deviation in Table 8 and empirical size in Table 9. We set $\delta=0.6, \gamma=0$, and ran the simulations for $\rho=0, .5, .75$, $-.5, \tau=.5,1$ and 2 and all combinations of the deterministic components, but to facilitate readability we exclude $D T c$, for which Assumption 2 is not met, and $\rho=0.5$, because the results are not much different than for $\rho=0.75$. In Table 7 and in Table 9 we also exclude results for $\tau=2$ and $\tau=0.5$ because, as RH also found, they do not vary much, while in Table 8 we exclude $D T b$ and $D T d$ because the deterministic trend is so strong that the standard deviation is too small to indicate any effect.

The correlation $\rho$ is a potential source of bias in OLS, but the effect, presented in Table 7, is minimal, mainly visible in small samples, and almost only for $D T a$, which has the lowest rate of convergence. In the simulations of $\mathrm{RH}$ a small fraction of the bias of $\widetilde{\nu}$ passed to $\widehat{\nu}_{F}$, but here the preliminary estimate of $\nu$ is so precise, due to the faster rate of convergence, that basically no bias is incurred at this stage. Altering $\tau$ only affects dispersion: in Table 8 sample variance increases with $\tau$ for given $\rho$ and $D T$. The low impact of changes of $\rho$ on precision is also important because it leaves empirical size nearly unaffected: it is not surprising that on average the best approximation to the nominal size is for $\rho=0$, but even with the rather extreme $\rho=0.75$ the effect on empirical size is often much less than 0.01 .

\section{Empirical analysis of the PPP hypothesis.}

We apply our methodology to analyse the Purchasing Power Parity (PPP) hypothesis in three US cities: New York, Boston and Philadelphia. The PPP hypothesis indicates that arbitrage should induce prices of the same items to be the same in different places, and to react together to shocks affecting one of the two: if $y_{t}$ is the local price and $x_{t}$ is price of the same good elsewhere converted to the local currency (both prices being in logarithms), then $\mu_{11}=0, \nu=1$ and $\gamma<1$ in (8) and (9). Strictly speaking, cointegration is not prescribed, but in practise this is necessarily the case because it is generally acknowledged that $\delta \geq 1$ (a lower level of $\delta$ would imply antipersistence of inflation); empirical experience of positive inflation rates also suggests a deterministic component of order about $\phi_{2 \ddagger}=1.5$, i.e. a linear trend.

The intuition behind PPP is easy to grasp and in line with common sense. The implications for both economic theory and policy advice are important, because implicitly it can also be interpreted as indicating market integration, 
so it is not surprising that it has been widely analysed in the applied economic literature. The empirical failure of the PPP hypothesis, at least as a short run phenomenon, is well documented: it can be due to the use of price indices, rather than effective prices, their differences reflecting the difference in preferences of economic agents. In addition, the arbitrage effect may be reduced and delayed by the cost of actively searching on the neighbour market and of eventually shipping the good to the home market. The latter argument also suggests that some deviation should be allowed, at least in the short run, and the PPP model then quickly become a classical case-study for cointegration: Corbae and Ouliaris (1988) and Johansen and Juselius (1992) assuming $(\gamma, \delta)=(0,1)$. They followed two different approaches: Corbae and Ouliaris tested for a unit root with a Dickey-Fuller statistic on the difference $y_{t}-x_{t}$, while Johansen and Juselius first estimated the potential cointegrating vector and then tested the restriction. In both the cases the joint restrictions $\nu=1, \gamma=0$ were rejected. The PPP hypothesis largely remains a puzzle in cointegration analysis, evidence being still dubious.

The restriction on $\gamma$ imposed by the $(\gamma, \delta)=(0,1)$ paradigm is stronger than economic theory implies, disregarding a wide class of mean- (or conditionalmean- ) reverting processes that are indexed by a different $\gamma$. Allowing for $0<$ $\gamma<1$, semiparametric fractional cointegration analysis of PPP was carried out by Cheung and Lai (1993), who estimated $\nu$ by OLS and $\gamma$ by log periodogram regression. They discussed the existence of a cointegrating relation but did not test $\nu=1$. The GLS methodology presented in the previous sections on the other hand allows for a more efficient estimation and testing of $\nu$, but the trend must then be taken explicitly into account.

We employ logged quarterly price indices of Boston, New York and Philadelphia for 1950(1) through 2003 (3), so that $n=215$. The data were collected from Datastream and have codes USCPBOMAF, USCPNYMAF, USCPPHMAF; the sampling frequency was intended to be monthly, but for several years the data were only collected every second or third month both for Boston and Philadelphia: for each city we produced quarterly data by averaging. Data were then normalised, dividing each series by the first observation, and logarithms are finally taken. The normalisation is introduced to make the series visually comparable: as seen in Figure 1, they are dominated by a long-term deterministic component, to the extent that the three paths cannot be clearly distinguished. Thus we also present, in Figure 2, annualised first differences, corresponding to inflation rate. Our assumption is that long term inflation remained stable over the whole period: phases of high inflation, such as those observed after the two oil shocks, are still possible, but on adopting a very long perspective they seem to be temporary (albeit still persistent) phenomena rather than substantial, non-reverting breaks. As Diebold and Inoue (2001) showed, allowing random, occasional breaks, and a long enough time perspective, long memory can be a convenient way to describe the data. On the other hand an analysis that takes explicit account of apparent structural breaks might be more satisfactory. Inspection of the figures, especially with respect to inflation, seems 
indeed to suggest a common stochastic trend, the three indices reacting to the most relevant events, including the oil shocks.

We analyse cointegration pairwise, denoting the three cases Bo-NY, Ph-NY and Ph-Bo for New York and Boston, New York and Philadelphia, and Boston and Philadelphia, respectively. Of course if two pairs are both cointegrated the third one will be too and it is then redundant, but considering all three is sensible, especially in the preliminary phase of semiparametric analysis, where the estimates are robust to model misspecification but inefficient. The distinction between explanatory and dependent variables has no econometric implications in our framework, but we take New York as $x_{t}$ throughout, and Boston as $x_{t}$ in the $\mathrm{Ph}-\mathrm{Bo}$ model. The nominal size for the tests is set at $5 \%$.

We first tested the usual $\gamma=0, \delta=1$ framework. Though inflation is sometimes modelled as an $I(1)$ process, it is usually taken to be $I(0)$, implying $\delta=1$, as this is consistent with a monetary policy model in which the central bank aims to stabilise the growth rate of prices in the medium-run, and as it is often supported by empirical tests. We tested $\phi=1$ by the augmented DickeyFuller test, allowing for a constant in the AR model in levels (thus a linear trend in the (9)), with an $\mathrm{AR}(4)$ selected by sequential testing. We applied the same procedure (without an intercept) to the first differences with $\delta=2$ in mind but overall our results support $\delta=1$. The vector AR for the Johansen procedure was determined by inspecting the sequential likelihood ratio test (LR), the Schwarz (SC) and the Hannan and Quinn (HQ) statistics, these pointing in general to 5 lags. Using the procedure of Johansen (1991) cointegration was rejected in all the three models.

Evidence against PPP is often interpreted as indication of a lack of integration between markets, and cannot be explained by trade or cultural barriers, regulations or exchange rate instability in the present case. Since naive inspection of the data is suggestive of cointegration we thus investigate whether this can emerge in a fractional framework. In particular, we first examine the existence of a cointegrating relationship, in such a way as to avoid the consequences of misspecification of high frequency behaviour. The robust, but inefficient, estimates of $\gamma, \delta, \nu$ that are involved will also provide a comparison with the more efficient ones subsequently obtained. The results are presented in Table 10.

We estimated $\delta_{x}$ and $\delta_{y}$, the orders of integration of $x_{t}$ and of $y_{t}$, by means of the local Whittle estimates, $\widetilde{\delta}_{x}$ and $\widetilde{\delta}_{y}$, as described in Robinson (1995) but, in view of the anticipated nonstationarity we applied the method to first differences, then adding back 1. Any deterministic linear trend is thereby removed too. The bandwidth was $m=0.24 n^{4 / 5}=16$, which is approximately MSE - optimal when the process is a $\operatorname{FARIMA}(1, \mathrm{~d}, 0)$ with $\operatorname{AR}$ coefficient 0.5 (see Henry and Robinson (1996)). The estimates of $\delta$ were 1.53 (NY), 1.45 (Bo) and $1.40(\mathrm{Ph})$. On the basis of the statistics $\widetilde{T}_{y x}$ and $\bar{T}_{y x}$ of Robinson and Yajima (2001) the hypothesis $\delta_{y}=\delta_{x}$ was rejected at $5 \%$ for Ph-NY though the statistic depends on a trimming number, and if this is not large enough the rejection could be due to the presence of cointegration. In the other two cases equality of the orders was not rejected. We then computed the Hausman type statistics 
$\bar{H}_{y}$ and $\bar{H}_{x}$ for no-cointegration of Marinucci and Robinson (2001, equation (30)) rejecting in case of Ph-NY and of Ph-Bo but not for Bo-NY. This seeming inconsistency could reflect a type two error: in Marinucci and Robinson's Monte Carlo experiment the power of a comparable case was only about $50 \%$. Note also that for Bo-NY the more efficient memory parameter estimate $\widetilde{\delta}_{*}$ used in the test does not lie between the individual estimates.

For each of the two pairs, $\bar{\nu}$ was computed and in each case found to be very close to 1 . No-intercept OLS accords with economic theory, and the preliminary Johansen analysis suggested that the level of persistence $\gamma$, even if less than $\delta$, may be between between $1 / 2$ and 1 . The possibility of such a high $\gamma$ would suggest estimating it from first difference of the OLS residuals $\bar{v}_{1 t}=y_{t}-\bar{\nu} x_{t}$. On the basis of $\widetilde{\gamma}$, the local Whittle estimate of $\gamma,(25)$ is satisfied in each case but only barely in the case Bo-NY; point estimates were also below 1 for $\mathrm{Ph}-\mathrm{NY}$ and $\mathrm{Ph}-\mathrm{Bo}$, again in accordance with economic theory, while it was just above 1 for Bo-NY.

Our semiparametric analysis suggests that the PPP model could be reasonable for the three cities in fractional context, the rejection of the $(\gamma, \delta)=(0,1)$ version being due to the high persistence of deviations from the long-run relation. We then proceeded to a parametric analysis along the lines described in Section 4 with the aim of ultimately testing $\nu=1$.

We consider the $\mathrm{Ph}-\mathrm{NY}$ and Bo-NY relations only, the results appearing in Table 11. In (23) and (24) we took $g_{1}(t)=0, g_{2}(t)=t$. Using the previously obtained estimates of $\gamma, \delta$ (pooled), $\nu$, and denoting by $\widetilde{\mu}_{21}$ the estimate of $\mu_{21}$ obtained by OLS regression of $x_{t}$ on $t$, we formed the residuals $\widetilde{v}_{1 t}=y_{t}-$ $x_{t} \bar{\nu}, \widetilde{v}_{2 t}=x_{t}-\widetilde{\mu}_{21} t$, and then took $\widetilde{\gamma}$ and $\widetilde{\delta}$ fractional differences respectively, labelling them as $\bar{u}_{1 t}=\widetilde{v}_{1 t}(\widetilde{\gamma}), \bar{u}_{2 t}=\widetilde{v}_{2 t}(\widetilde{\delta})$. We then determined the structure $B(L ; h)$ using the LR, SC and HQ procedures on the series $\bar{u}_{1 t}, \bar{u}_{2 t}$, concluding in favour of a vector $\operatorname{AR}(4)$. Since the coefficients of the second and third lags are small, we took them to be zero, representing the short term dynamics by, in effect, an $\mathrm{AR}(1)$ combined with a quarterly effect.

Now define, for given $c, d, \widetilde{u}_{t}(c, d)=\left(\widetilde{v}_{1 t}(c), \widetilde{v}_{2 t}(d)\right)^{\prime}$ and $\widetilde{\varepsilon}_{t}(c, d)=B(L ; \widetilde{\theta}(c, d)) \widetilde{u}_{t}(c, d)$ where $\widetilde{\theta}(c, d)$ indicates the OLS estimates of the AR coefficients for given $c, d$, dropping $\widetilde{u}_{1}(c, d)$ because this ${ }^{6}$ term is not differenced at all. We then take $(\widehat{\gamma}, \widehat{\delta})=\arg \min n^{-1} \sum_{t} \widetilde{\varepsilon}_{t}(c, d) \widetilde{\varepsilon}_{t}^{\prime}(c, d)$, and $\widehat{\theta}=\widetilde{\theta}(\widehat{\gamma}, \widehat{\delta}), \widehat{\Omega}=n^{-1} \sum_{t} \widetilde{\varepsilon}_{t}(\widehat{\gamma}, \widehat{\delta}) \widetilde{\varepsilon}_{t}^{\prime}(\widehat{\gamma}, \widehat{\delta})$. Hypothesis on $\gamma, \delta, \theta$ can be tested with a likelihood ratio statistic.

This analysis confirmed restrictions on the $\operatorname{AR}(4)$ model for $u_{t}$, while $\widehat{\gamma}$ and $\widehat{\delta}$ were similar to the semiparametric estimates. We strongly rejected the joint hypothesis that $\delta=1, \gamma=0$. On the other hand the hypothesis $\gamma=1$ was rejected against the alternative $\gamma \neq 1$. We then applied the time domain GLS procedure to estimate $\nu$ : the estimates $\widehat{\nu}$ were in both cases close to 1 , and the null hypothesis was not rejected.

In Figure 3 and 4 we plotted the GLS residuals $y_{t}-\widehat{\nu} x_{t}$ (where $\widehat{\nu}$ is the GLS estimate of $\nu$ ) and the restricted residuals $y_{t}-x_{t}$ (assuming $\nu=1$ ), respectively. In both cases the residual series only occasionally cross 0 (the value that we assumed to be the mean the disturbances). The patterns of GLS and restricted 
residuals are very similar, but autocorrelation appears to be a little stronger for the latter: iterating the parametric procedure, the estimates $\widehat{\gamma}$ using GLS residuals $y_{t}-\widehat{\nu} x_{t}$ are 0.65 for the pair Ph-NY and 0.69 for the pair Bo-NY, while using the restricted residuals they are both 0.73 .

As a final small exercise we investigated the role of the deterministic trend in increasing precision in the estimation of $\nu$, bearing in mind the findings of the Monte Carlo exercise. We thus also computed the GLS estimate of RH, using detrended data. The estimates $\left((1,0)\left(b^{*}\right)^{-1}(1,0)^{\prime}\right)^{1 / 2}$ (see Corollary 4) were 0.0107 in case of $\mathrm{Ph}-\mathrm{NY}$, and 0.0151 in case of Bo-NY, whereas the $\left(b^{*}\right)^{-1 / 2}$ defined from $\mathrm{RH}$ for the detrended data were more than twice the size, being 0.0304 and 0.0415 respectively.

\section{Appendix}

Proof of Theorem 1 Define $g(t)=\left(t^{\phi_{1}-\frac{1}{2}}, \ldots, t^{\phi_{r}-\frac{1}{2}}\right)^{\prime}, D_{g n}=\operatorname{diag}\left\{n^{\phi_{1}}, \ldots, n^{\phi_{r}}\right\}$, for $0<\phi_{1}<\ldots<\phi_{r}$. For $d \geq 0$ we have

$n^{-d}\left(D_{g n}^{-1} \otimes I_{2}\right) \sum_{t} g(t) \otimes u_{t}(-d) \rightarrow_{d} \int_{0}^{1} g(r) \otimes d(\bar{W}(r ; d+1), \widetilde{W}(r ; d+1))^{\prime}$,

where $\otimes$ denotes Kronecker product. For $d>\frac{1}{2}$ (112) follows from Theorem 1 of Marinucci and Robinson (2000) (hereafter MR), and the continuous mapping theorem. For $0 \leq d \leq \frac{1}{2}$ (112) follows from a central limit theorem; note that the right side of (112) is in any case a $2 r$-dimensional normal variate. We have

$$
x_{t}=u_{2 t}(-\delta)+s_{x t},
$$

where

$$
s_{x t}=\sum_{j=1}^{p_{2}} \mu_{2 j} t^{\phi_{2 j}-\frac{1}{2}}
$$

From (112),

$$
\begin{gathered}
n^{-\phi_{2 \ddagger}-\delta} \sum_{t} s_{x t} u_{2 t}(-\delta)=n^{-\phi_{2 \ddagger}-\delta} \mu_{2 \ddagger} \sum_{t} t^{\phi_{2 \ddagger}-\frac{1}{2}} u_{2 t}(-\delta)+o_{p}(1) \\
\rightarrow_{d} \Phi_{2} .
\end{gathered}
$$

By Theorem 1 of MR and the continuous mapping theorem

$$
n^{-2 \delta} \sum_{t} u_{2 t}^{2}(-\delta) \rightarrow_{d} \Phi_{1}
$$

and by integral approximation

$$
\begin{aligned}
n^{-2 \phi_{2 \ddagger}} \sum_{t} s_{x t}^{2} & =n^{-2 \phi_{2 \ddagger}} \mu_{2 \ddagger}^{2} \sum_{t} t^{2 \phi_{2 \ddagger}-1}+o(1) \\
& \rightarrow \Phi_{3} .
\end{aligned}
$$


Thus

$$
k_{n}^{-2} M_{x x} \rightarrow_{d} \Phi_{1}^{*}+2 \Phi_{2}^{*}+\Phi_{3}^{*} .
$$

Next, the numerator of $\bar{\nu}-\nu$ is

$$
a=\sum_{t}\left(s_{x t}+u_{2 t}(-\delta)\right)\left(s_{y t}+u_{1 t}(-\gamma)\right)
$$

where

$$
s_{y t}=\sum_{j=1}^{p_{1}} \mu_{1 j} t^{\phi_{1 j}-\frac{1}{2}} .
$$

Integral approximation gives

$$
\begin{aligned}
n^{-\phi_{1 \dagger}-\phi_{2 \ddagger}} \sum_{t} s_{x t} s_{y t} & =n^{-\phi_{1 \dagger}-\phi_{2 \ddagger}} \mu_{1 \dagger} \mu_{2 \ddagger} \sum_{t} t^{\phi_{1 \dagger}+\phi_{2 \ddagger}-1}+o(1) \\
& \rightarrow \Psi_{6}
\end{aligned}
$$

and (112) gives

$$
\begin{aligned}
& n^{-\gamma-\phi_{2 \ddagger}} \sum_{t} s_{x t} u_{1 t}(-\gamma) \rightarrow_{d} \Psi_{4} . \\
& n^{-\delta-\phi_{1 \dagger}} \sum_{t} s_{y t} u_{2 t}(-\delta) \rightarrow_{d} \Psi_{5},
\end{aligned}
$$

To deal with $b=\sum_{t} u_{1 t}(-\gamma) u_{2 t}(-\delta)$, we make use of results of RM. From Lemmas 4.1, 4.2 and 5.1 of RM (see also their Propositions 6.1 and 6.2) we have

$$
\begin{aligned}
n^{-1} b \rightarrow_{p} \Psi_{1}, \quad \text { if } \gamma+\delta<1, \\
\frac{n^{-1}}{\log n} \rightarrow_{p} \Psi_{2}, \quad \text { if } \gamma+\delta=1, \gamma>0 .
\end{aligned}
$$

From Lemmas 4.3, 4.5 and 5.1 of RM, $b=O_{p}\left(n^{\delta}\right)$ for $\gamma=0, \delta \geq 1$, but since $\phi_{1 \dagger}>0$ it follows that in this case $a=o_{p}\left(n^{\delta+\phi_{1 \ddagger}}\right)$ so $\Psi_{5}$ will dominate. Finally

$$
n^{-\gamma-\delta} b \rightarrow_{d} \Psi_{3}, \quad \gamma+\delta>1, \gamma>0,
$$

where Theorem 1 of MR and the continuous mapping theorem covers the case $\gamma>\frac{1}{2}$, and Lemmas 4.5 and 5.1 of MR the case $\gamma \leq \frac{1}{2}$ (RM discuss the problem of representation of $\Psi_{3}$ in this case). It follows that

$$
\ell_{n}^{-1} a \rightarrow_{d} \Psi_{1}^{*}+\Psi_{2}^{*}+\Psi_{3}^{*}+\Psi_{4}^{*}+\Psi_{5}^{*}+\Psi_{6}^{*},
$$

noting that in case $\gamma+\delta=1, \gamma>0, n \log n$ dominates $n=n^{\gamma+\delta}$, and dominates $n^{\gamma+\phi_{2 \ddagger}}, n^{\delta+\phi_{1 \dagger}}, n^{\phi_{1 \dagger}+\phi_{2 \ddagger}}$ if and only if $\delta \geq \phi_{2 \ddagger}, \gamma \geq \phi_{1 \dagger}, \phi_{1 \dagger}+\phi_{2 \ddagger} \leq 1$ respectively.

Proof of Theorem 2 The proof that

$$
B_{n}^{-1} M_{x_{+} x_{+}} B_{n}^{-1} \rightarrow_{d} \Phi
$$


straightforwardly uses results employed in showing (112), and is omitted. We are left to consider

$$
M_{x_{+} v}=\sum_{t} x_{+t}\left\{u_{1 t}(-\gamma)+s_{1 t}\right\}=\sum_{t}\left(u_{2 t}(-\delta)+s_{x t}, g_{1}^{\prime}(t)\right)^{\prime}\left\{u_{1 t}(-\gamma)+s_{1 t}\right\} .
$$

By again employing results from the proof of Theorem 1,

$$
D_{1 n}^{-1}(\gamma) \sum_{t} g_{1}(t) u_{1 t}(-\gamma) \rightarrow_{d} \Psi_{8}
$$

while

$$
D_{1 n}^{-1}(\gamma) \sum_{t} g_{1}(t) s_{1 t} \rightarrow 0
$$

because, for $j>p_{11}$ and $i+1 \ldots p_{11}$

$$
n^{-\gamma-\phi_{1 i}} \sum_{t} t^{\phi_{1 i}+\phi_{1 j}}=O\left(n^{\phi_{1 j}-\gamma}\right)=o(1)
$$

because $\gamma>\phi_{1 j}$ for $j>p_{11}$. Next,

$$
\sum_{t} s_{x t} s_{1 t}=O\left(n^{\phi_{2 \ddagger}+\phi_{1, p_{11}+1}}\right)=o\left(n^{\phi_{2 \ddagger}+\gamma}\right) .
$$

Then from the proof of Theorem 1,

$$
m_{n}^{-1} \sum_{t}\left\{u_{2 t}(-\delta)+s_{x t}\right\} u_{1 t}(-\gamma) \rightarrow_{d} \Psi_{1}^{* *}+\Psi_{2}^{* *}+\Psi_{3}^{* *}+\Psi_{4}^{* *}+\Psi_{7}^{* *}
$$

with $\Phi_{7}^{* *}$ coming from MR Theorem 1 and Kurtz and Protter (1991), see also MR Propositions 6.3 and 6.4 (unlike in Theorem 1, this contribution is not always dominated).

Further, we may obtain the joint result

$$
C_{n}^{-1} B_{n}^{-1} M_{x_{+} v} \rightarrow_{d} \Psi \text {. }
$$

From the commutativity properties of diagonal matrices

$$
\widetilde{\nu}_{+}-\nu=B_{n}^{-1}\left(B_{n}^{-1} M_{x_{+} x_{+}} B_{n}^{-1}\right)^{-1} C_{n}\left(C_{n}^{-1} B_{n}^{-1} M_{x_{+} v}\right)
$$

so the proof is completed by application of (128) and (136).

Proof of Theorem 3 Consider the case of white noise $u_{t}$. From (8), (9), (90), (98) and (99) we can write

$$
\widehat{\nu}_{++}(\gamma, \delta, \theta)-\nu=b(\gamma, \delta, \theta)^{-1}\{e(\gamma, \delta, \theta)+f(\gamma, \delta, \theta)\},
$$

where

$$
e(\gamma, \delta, \theta)=\sum_{t} w_{t}(\gamma, \delta, \theta) \Omega^{-1} u_{t}, \quad f(\gamma, \delta, \theta)=\sum_{t} w_{t}(\gamma, \delta, \theta) \Omega^{-1} \widetilde{s}_{t},
$$


where $\widetilde{s}_{t}=\left(\widetilde{s}_{1 t}, \widetilde{s}_{2 t}\right)^{\prime}=\left(\Delta^{\gamma} s_{1 t}, \Delta^{\delta} s_{2 t}\right)^{\prime}$. From Lemma 1 of Robinson (2003), with $v_{t}=t^{c}$,

$$
v_{t}(d)=\frac{\Gamma(c+1)}{\Gamma(c-d+1)} t^{c-d}+O\left(t^{c-m-1}\right),
$$

where $m$ is the integer such that $d-1<m \leq d$. Then

$$
\begin{aligned}
\sum_{t} \Delta^{\gamma} g_{2}^{\prime}(t) \mu_{2} \widetilde{s}_{1 t} & =O\left(\sum_{t} t^{\phi_{2 \ddagger}-2 \gamma+\phi_{1, p_{11}+1}-1}\right) \\
& =O\left(n^{\phi_{2 \ddagger}+\phi_{1, p_{11}+1}-2 \gamma}\right), \\
\sum_{t} \Delta^{\gamma} g_{1 j}(t) \widetilde{s}_{1 t} & =O\left(\sum_{t} t^{\phi_{1 j}-2 \gamma+\phi_{1, p_{11}+1}-1}\right) \\
& =O\left(n^{\phi_{1 j}+\phi_{1, p_{11}+1}-2 \gamma}\right), \quad j=1, \ldots, p_{11}, \\
\sum_{t} \Delta^{\delta} g_{2 j}(t) \widetilde{s}_{1 t} & =O\left(\sum_{t} t^{\phi_{2 j}-\gamma-\delta+\phi_{1, p_{11}+1}-1}\right) \\
& =O\left(n^{\phi_{2 j}+\phi_{1, p_{11}+1}-\gamma-\delta}\right), \quad j=1, \ldots, p_{21},
\end{aligned}
$$

and similarly $\sum_{t} \Delta^{\gamma} g_{2}^{\prime}(t) \mu_{2} \widetilde{s}_{2 t}=O\left(n^{\phi_{2 \ddagger}+\phi_{2, p_{21}+1}-\gamma-\delta}\right), \sum_{t} \Delta^{\gamma} g_{1 j}(t) \widetilde{s}_{2 t}=O\left(n^{\phi_{1 j}+\phi_{2, p_{21}+1}-\gamma-\delta}\right)$ for $j=1 \ldots p_{11}, \sum_{t} \Delta^{\delta} g_{2 j}(t) \widetilde{s}_{2 t}=O\left(n^{\phi_{2 j}+\phi_{2, p_{21}+1}-2 \delta}\right)$ for $j=1 \ldots p_{21}$.

On the other hand, much as in the proof of Theorem 1,

$\sum_{t} u_{2 t}(\gamma-\delta) \widetilde{s}_{1 t}=O_{p}\left(n^{\delta-2 \gamma+\phi_{1, p_{11}+1}+\frac{1}{2}}\right), \sum_{t} u_{2 t}(\gamma-\delta) \widetilde{s}_{2 t}=O_{p}\left(n^{-\gamma+\phi_{2, p_{21}+1}+\frac{1}{2}}\right)$.

It straightforwardly follows that

$$
D_{n}^{-1} f(\gamma, \delta, \theta) \rightarrow 0 .
$$

From (139) and routine arguments

$$
n^{\frac{1}{2}} D_{i n}^{-1}(-d) \Delta^{d} g_{i}([r n]) \rightarrow \Lambda_{i}(d) \frac{g_{i}(r)}{r^{d}}, \quad r \in(0,1], \quad d \geq 0, i=1,2 .
$$

From this and MR Theorem 1

$$
n^{\frac{1}{2}} D_{n}^{-1} w_{[r n]}(\gamma, \delta) \Rightarrow Q(r), \quad r \in(0,1] .
$$

Thus from the continuous mapping theorem and Kurtz and Protter (1991)

$$
D_{n}^{-1} b(\gamma, \delta, \theta) D_{n}^{-1} \Rightarrow \int_{0}^{1} Q(r) Q^{\prime}(r) d r, \quad D_{n}^{-1} e(b, \delta, \theta) \Rightarrow \int_{0}^{1} Q(r) d W(r)
$$

to complete the proof for $\widehat{\nu}_{++}(\gamma, \delta, \theta)$. The application of Assumption 3 to prove the Theorem for the remaining quantities in (101), in the white noise $u_{t}$ case, is straightforward, and thus omitted. 
A good deal of the proof detail in $\mathrm{RH}$ is concerned with justifying the general short memory autocorrelation in $u_{t}$ described in Section 4. It is clearly unnecessary to repeat this for our extended estimate, and it suffices merely to consider the implications for the deterministic components we have introduced. These are somewhat different from the treatment of stochastic trends. Consider the quantity

$$
\widetilde{e}(\gamma, \delta, \theta)=\sum_{j} F_{\widetilde{w}(\gamma, \delta)}\left(\lambda_{j}\right) f\left(\lambda_{j}\right)^{-1} F_{u}\left(-\lambda_{j}\right),
$$

where

$$
F_{\widetilde{w}(\gamma, \delta)}(\lambda)=\frac{1}{(2 \pi n)^{\frac{1}{2}}} \sum_{t} \widetilde{w}_{t}(\gamma, \delta) e^{i t \lambda}, F_{u}(\lambda)=\frac{1}{(2 \pi n)^{\frac{1}{2}}} \sum_{t} u_{t} e^{i t \lambda},
$$

with $\widetilde{w}_{t}(c, d)$ defined like $w_{t}(c, d)$ in (85) but with $\Delta^{c} x_{t}$ replaced by $\Delta^{c} s_{x t}$. Denote by $\psi_{L}(\lambda)=\sum_{\ell=-L}^{L} \psi_{\ell}(1-|\ell| / L) e^{-i \ell \lambda}$ the Cesaro sum, to $L$ terms, of the Fourier series of $f(\lambda)^{-1}$. Define $\widetilde{D}_{n}$ like $D_{n}$ but with $n^{\max \left(\delta, \phi_{2 \ddagger}\right)}$ replaced by $n^{\phi_{2 \ddagger}}$ and

$$
\widehat{e}(\gamma, \delta, \theta)=\sum_{j} F_{\widetilde{w}(\gamma, \delta)}\left(\lambda_{j}\right) \psi_{L}\left(\lambda_{j}\right) F_{u}\left(-\lambda_{j}\right) .
$$

Then $\widetilde{D}_{n}^{-1}\{\widetilde{e}(\gamma, \delta, \theta)-\widehat{e}(\gamma, \delta, \theta)\}$ has mean zero and covariance matrix

$$
\begin{aligned}
& \frac{1}{2 \pi n} \int_{-\pi}^{\pi}\left\{\widetilde{D}_{n}^{-1} \sum_{j} F_{\widetilde{w}(\gamma, \delta)}\left(\lambda_{j}\right)\left\{f\left(\lambda_{j}\right)^{-1}-\psi_{L}\left(\lambda_{j}\right)\right\} \sum_{s} e^{i\left(\lambda_{j}-\lambda\right) s}\right\} f(\lambda) \\
& \times\left\{\widetilde{D}_{n}^{-1} \sum_{j} F_{\widetilde{w}(\gamma, \delta)}\left(-\lambda_{j}\right)\left\{f\left(-\lambda_{j}\right)^{-1}-\psi_{L}\left(-\lambda_{j}\right)\right\} \sum_{j} e^{i\left(\lambda-\lambda_{j}\right) s}\right\} d \lambda .(151)
\end{aligned}
$$

Using the properties of the complex exponential function, this has norm bounded by a constant times

$$
\sum_{j}\left\|\widetilde{D}_{n}^{-1} F_{\widetilde{w}(\gamma, \delta)}\left(\lambda_{j}\right)\left\{f\left(\lambda_{j}\right)^{-1}-\psi_{L}\left(\lambda_{j}\right)\right\}\right\|^{2} \leq \varepsilon^{2} \sum_{j}\left\|\widetilde{D}_{n}^{-1} F_{\widetilde{w}(\gamma, \delta)}\left(\lambda_{j}\right)\right\|^{2}
$$

for arbitrary $\varepsilon>0$, on choosing $L$ large enough and noting the continuity of $f(\lambda)^{-1}$. For any sequence $c_{t}$

$$
n^{-1} \sum_{j}\left|\sum_{t} c_{t} e^{i t \lambda_{j}}\right|^{2}=\sum_{t} c_{t}^{2},
$$

so applying again (147), it straightforwardly follows that $(152)=O\left(\varepsilon^{2}\right)$. Thus

$$
\begin{aligned}
\widehat{e}(\gamma, \delta, \theta)= & \sum_{\ell=-L}^{L} \sum_{t}^{\prime} \widetilde{w}_{t}(\gamma, \delta) \psi_{\ell}\left(1-\frac{|\ell|}{L}\right) u_{t-\ell} \\
& +\sum_{\ell=1}^{L} \sum_{t=1}^{\ell} \widetilde{w}_{t}(\gamma, \delta) \psi_{\ell}\left(1-\frac{\ell}{L}\right) u_{t-\ell}, \\
& +\sum_{\ell=-L}^{-1} \sum_{t=n-\ell+1}^{n} \widetilde{w}_{t}(\gamma, \delta) \psi_{\ell}\left(1+\frac{\ell}{L}\right) u_{t-\ell},
\end{aligned}
$$


where $\sum_{t}^{\prime}=\sum_{1 \leq t, t-\ell \leq n}$. The sums over $t$ in (155) and (156) include only $\ell$ terms, and, with $L$ fixed and $n \rightarrow \infty$, will turn out to be dominated by (154). To deal with this, note from boundedness of $f$ that for $c>0, d>0$ and any $j$,

$$
\sum_{t}\left\{\Delta^{d} t^{c}-\frac{\Gamma(c+1) t^{c-d}}{\Gamma(c-d+1)}\right\} u_{t-j}
$$

has mean zero and variance bounded by a constant times

$$
\sum_{t}\left\{\Delta^{d} t^{c}-\frac{\Gamma(c+1) t^{c-d}}{\Gamma(c-d+1)}\right\}^{2}=O\left(\sum_{t} t^{2(c-m-1)}\right)
$$

where $m$ is as described after (139). Then (158) is $O(1)$ for $2 c-2 m<1, O(\log n)$ for $2 c-2 m=1$ and $O\left(n^{2 c-2 m+1}\right)$ for $2 c-2 m>1$. It follows that we may replace $\widetilde{D}_{n}^{-1} \widehat{e}(\gamma, \delta, \theta)$ by

$$
\widetilde{D}_{n}^{-1} \sum_{\ell=-L}^{L} \sum_{t}^{\prime} \widehat{w}_{t}(\gamma, \delta) \psi_{\ell}\left(1-\frac{|\ell|}{L}\right) u_{t-\ell}
$$

where

$\widehat{w}_{t}(\gamma, \delta)=\left[\begin{array}{ccc}\mu_{2 \ddagger} \frac{\Gamma\left(\phi_{2 \ddagger}+\frac{1}{2}\right)}{\Gamma\left(\phi_{2 \ddagger}-\gamma+\frac{1}{2}\right)} t^{\phi_{2 \ddagger}-\gamma-\frac{1}{2}} & \left\{\Lambda_{1}(\gamma) g_{1}(t)\right\}^{\prime} t^{-\gamma} & 0 \\ 0 & 0 & \left\{\Lambda_{2}(\delta) g_{2}(t)\right\}^{\prime} t^{-\delta}\end{array}\right]^{\prime}$,

the above arguments indicating that other contributions from the top left hand element of $\widetilde{w}_{t}(\gamma, \delta)$ can be neglected. The asymptotic normality, for fixed $L$, of (159) follows standardly (see e.g. Hannan, 1970, Chapter 7), whence Bernstein's lemma, with $L \rightarrow \infty$, completes the central limit theorem for $\widetilde{D}_{n}^{-1} \widetilde{e}(\gamma, \delta, \theta)$. The proof that we can neglect contributions from $s_{1 t}$ and $s_{2 t}$ follows much as above, as does, using also $\mathrm{RH}$, the limiting behaviour of $D_{n}^{-1} b(\gamma, \delta, \theta) D_{n}^{-1}$. The proof that we can replace $\gamma, \delta, \theta$ by $\widehat{\gamma}, \widehat{\delta}, \widehat{\theta}$ is lengthy but relies basically on $\mathrm{RH}$ and standard arguments to cope with the deterministic components. As in $\mathrm{RH}$, the proof for the 'time - domain' estimates is similar but slightly simpler, and is omitted. 


\section{References}

[1] CAMPOS, J., ERICSSON, N.R., HENDRY, D.F., 1996. Cointegration in the presence of structural breaks. Journal of Econometrics 70, 187-220.

[2] CHAN, N.H., TERRIN, N., 1995. Inference for unstable long-memory processes with applications to fractional unit root autoregressions. Annals of Statistics 23, 1662-1683.

[3] CHEN, W.W., HURVICH, C.M., 2003. Estimating fractional cointegration in the presence of polynomial trends. Journal of Econometrics 117, 95-121.

[4] CHEUNG, Y.W., LAI, K.S., 1993. A fractional cointegration analysis of purchasing power parity. Journal of Business and Economic Statistics 11, 103-112.

[5] CORBAE, D., OULIARIS, S., 1988. Cointegration and tests of purchasing power parity. Review of Economics and Statistics 70, 508-511.

[6] DIEBOLD, F.X., INOUE, A., 2001. Long memory and regime switching. Journal of Econometrics 105, 131-159.

[7] DOLADO, J., MARMOL, F., 1997. Efficient estimation of cointegrating relationships among higher order and fractionally integrated processes. Preprint.

[8] HANSEN, B.E., 1992. Efficient estimation and testing of cointegration vectors in the presence of deterministic trends. Journal of Econometrics 53, $87-121$.

[9] HANNAN, E.J., 1970. Multiple Time Series. Wiley, New York.

[10] HASSleR, U., MARMOL, F., VELASCO, C., 2002. Residual logperiodogram inference for long-run relationships. Preprint.

[11] HENRY, M., ROBINSON, P.M., 1996. Bandwidth choice in Gaussian semiparametric estimation of long-range dependence, in Athens Conference on Applied Probability and Time Series Analysis, Vol. II: Time Series Analysis (P. M. Robinson and M. Rosenblatt, eds), New York: Springer-Verlag, pp. $220-32$.

[12] HOFFMAN, D.L., RASCHE, R.H., 1991. Long run income and interest elasticities of of money demand in the United States. The Review of Economics and Statistics 73, 665-674.

[13] HUALDE, J., ROBINSON, P.M., 2001. Root-n-consistent estimation of weak fractional cointegration. Preprint.

[14] JEGANATHAN, P., 1999. On asymptotic inference in cointegrated time series with fractionally integrated errors. Econometric Theory 15, 583-621. 
[15] JOHANSEN, S., 1991. Estimation and hypothesis testing of cointegrating vectors in Gaussian vector autoregressive models. Econometrica 59, 15511580 .

[16] JOHANSEN, S., JUSELIUS, K., 1992. Testing structural hypotheses in a multivariate cointegration analysis of the PPP and the UIP for the UK. Journal of Econometrics 53, 211-244.

[17] KIM, C.S., PHILLIPS, P.C.B., 2000. Fully modified estimation of fractional cointegration models. Preprint.

[18] KURTZ, T.G., PROTTER, P., 1991. Weak limit theorems for stochastic integrals and stochastic differential equations. Annals of Probability 19, 1035-1070.

[19] MARINUCCI, D., ROBINSON, P.M., 2000. Weak convergence of multivariate fractional processes. Stochastic Processes and their Applications 86, 103-120.

[20] MARINUCCI, D., ROBINSON, P.M., 2001. Semiparametric fractional cointegration analysis. Journal of Econometrics 105, 225-247.

[21] OGAKI, M., PARK, J.Y., 1997. A cointegration approach to estimating preference parameters. Journal of Econometrics 82, 107-134.

[22] PERRON, P., CAMPBELL, J.Y., 1993. A note on Johansen's procedure when trends are present. Empirical Economics 18, 777-789.

[23] PHILLIPS, P.C.B., 1991a. Optimal inference in cointegrated systems. Econometrica 59, 283-306.

[24] PHILLIPS, P.C.B., 1991b. Spectral regression for cointegrated time series. In W.A. Barnett, J. Powell and G. Tauchen (eds.) Nonparametric and Semiparametric Methods in Econometrics and Statistics. Cambridge: Cambridge University Press, 413-435.

[25] PHILlIPS, P.C.B., HANSEN, B.E., 1990. Statistical inference in instrumental variables regression with I(1) processes. Review of Economic Studies $57,99-125$.

[26] ROBINSON, P.M., 1986. On the errors-in-variables problem for time series. Journal of Multivariate Analysis 19, 240-250.

[27] ROBINSON, P.M., 1994. Semiparametric analysis of long memory time series. Annals of Statistics 22, 515-539.

[28] ROBINSON, P.M., 1995. Gaussian semiparametric estimation of long range dependence. Annals of Statistics 23, 1630 - 1661.

[29] ROBINSON, P.M., 2003. Efficiency improvements in inference on stationary and nonstationary fractional time series. Preprint. 
[30] ROBINSON, P.M., HUALDE, J., 2003. Cointegration in fractional systems with unknown integration orders. Econometrica 71, 1727-1766.

[31] ROBINSON, P.M., MARINUCCI, D., 2000. The averaged periodogram for nonstationary vector time series. Statistical Inference for Stochastic Processes 3, 149-160.

[32] ROBINSON, P.M., MARINUCCI, D., 2001. Narrow band analysis of nonstationary processes. Annals of Statistics 29, 947-986.

[33] ROBINSON, P.M., MARINUCCI, D., 2003. Semiparametric frequency domain analysis of fractional cointegration. Time Series with Long Memory (P.M. Robinson, ed.). Oxford University Press, Oxford, 334-373.

[34] ROBINSON, P.M., YAJIMA, Y., 2002. Determination of cointegrating ranking fractional systems. Journal of Econometrics 106, 217-241.

[35] SÖDERLIND, P., VREDIN A., 1996. Applied cointegration analysis in the mirror of macroeconomic theory. Journal of Applied Econometrics 11, 363381.

[36] STOCK, J., WATSON, M.W., 1988. Testing for common trends. Journal of the American Statistical Society 83, 1097-1107.

[37] STOCK, J., WATSON, M.W., 1993. A simple estimator of cointegrating vectors in higher order integrated systems. Econometrica 61, 783-820.

[38] VELASCO, C., 2000. Gaussian semiparametric estimation of fractional cointegration. Preprint.

[39] VELASCO, C., ROBINSON, P.M., 2000. Whittle pseudo-maximum likelihood estimation for nonstationary time series. Journal of the American Statistical Association 95, 1229-1243.

[40] WEST, K.D., 1988. Asymptotic normality when regressors have a unit root. Econometrica 56, 1397-1417. 
TABLE 1

CONVERGENCE RATES (POWERS OF $n$ ) OF $\bar{\nu}$

\begin{tabular}{lllllll}
\hline \hline & $S T a$ & $S T b$ & $S T c$ & $S T d$ & $S T e$ & $S T f$ \\
\hline $0^{*}$ & 0.2 & 1.2 & 2 & $0.2^{\dagger}$ & 0.8 & 1.6 \\
$D T a$ & 1 & 1 & 1.5 & 1 & 1 & 1.5 \\
$D T b$ & 2 & 2 & 2 & 2 & 2 & 2 \\
$D T c$ & $\mathrm{X}$ & $\mathrm{X}$ & 0.5 & $\mathrm{X}$ & $\mathrm{X}$ & 0.5 \\
$D T d$ & 1 & 1 & 1 & 1 & 1 & 1 \\
\multicolumn{7}{c}{ *: Refers to the case of no trend, as in RM. }
\end{tabular}

${ }^{\dagger}$ : The rate is actually $n^{0.2} / \ln n$

$\mathrm{X}$ : Inconsistent

TABLE 2:

CONVERGENCE RATES (POWERS OF $n$ ) OF $\widetilde{\nu}$

\begin{tabular}{lllllll}
\hline \hline & $S T a$ & $S T b$ & $S T c$ & $S T d$ & $S T e$ & $S T f$ \\
\hline$D T a$ & 1.5 & 1.5 & 2 & 1.1 & 1.1 & 1.6
\end{tabular}

$\begin{array}{lllllll}D T b & 2.5 & 2.5 & 2.5 & 2.1 & 2.1 & 2.1\end{array}$

$\begin{array}{llll}\text { DTc } & 2 & 1.6\end{array}$

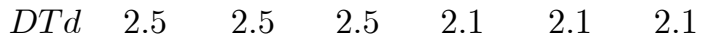

Note: convergence rates not reported when Assumption 2 is not met.

TABLE 3:

CONVERGENCE RATES (POWERS OF $n$ ) OF $\widehat{\nu}$

\begin{tabular}{lllllll}
\hline \hline & $S T a$ & $S T b$ & $S T c$ & $S T d$ & $S T e$ & $S T f$ \\
\hline$D T a$ & 1.5 & 1.5 & 2 & & 1.1 & 1.6 \\
$D T b$ & 2.5 & 2.5 & 2.5 & & 2.1 & 2.1 \\
$D T c$ & & & 2 & & & 1.6
\end{tabular}

\begin{tabular}{llllll}
$D T d$ & 2.5 & 2.5 & 2.5 & 2.1 & 2.1 \\
\hline
\end{tabular}

Note: convergence rates not reported when Assumption 2 or $\beta>0.5$ is not met. 
TABLE 4

MONTE CARLO BIAS FOR $\tau=1, \rho=0.5,1000$ REPLICATIONS.

\begin{tabular}{|c|c|c|c|c|c|c|c|c|c|c|c|c|c|}
\hline \multirow[b]{2}{*}{$S T$} & \multirow[b]{2}{*}{$D T$} & \multicolumn{4}{|c|}{$n=64$} & \multicolumn{4}{|c|}{$n=128$} & \multicolumn{4}{|c|}{$\overline{n=256}$} \\
\hline & & $\bar{\nu}$ & $\widetilde{\nu}$ & $\widehat{\nu}_{I}$ & $\widehat{\nu}_{F}$ & $\bar{\nu}$ & $\widetilde{\nu}$ & $\widehat{\nu}_{I}$ & $\widehat{\nu}_{F}$ & $\bar{\nu}$ & $\widetilde{\nu}$ & $\widehat{\nu}_{I}$ & $\widehat{\nu}_{F}$ \\
\hline \multirow{4}{*}{$a$} & $a$ & .0235 & .0009 & .0000 & .0000 & .0117 & .0003 & .0000 & .0000 & .0059 & .0001 & .0000 & .0000 \\
\hline & $b$ & .0004 & .0000 & .0000 & .0000 & .0001 & .0000 & .0000 & .0000 & .0000 & .0000 & .0000 & .0000 \\
\hline & $c$ & 1.0232 & .2980 & .1549 & .1634 & 1.0112 & .2515 & .1145 & .1201 & 1.0057 & .2076 & .0804 & .0841 \\
\hline & $d$ & .0194 & .0000 & .0000 & .0000 & .0097 & .0000 & .0000 & .0000 & .0049 & .0000 & .0000 & .0000 \\
\hline \multirow{4}{*}{$b$} & $a$ & .0247 & .0003 & .0001 & .0000 & .0121 & .0000 & .0000 & .0000 & .0060 & .0000 & .0000 & .0000 \\
\hline & $b$ & .0004 & .0000 & .0000 & .0000 & .0001 & .0000 & .0000 & .0000 & .0000 & .0000 & .0000 & .0000 \\
\hline & $c$ & 1.0912 & .0176 & -.0002 & -.0040 & 1.0505 & .0048 & .0006 & -.0007 & 1.0347 & .0006 & .0004 & .0002 \\
\hline & $d$ & .0194 & .0000 & .0000 & .0000 & .0097 & .0000 & .0000 & .0000 & .0049 & .0000 & .0000 & .0000 \\
\hline \multirow{4}{*}{$c$} & $a$ & .0016 & -.0017 & -.0003 & -.0006 & .0005 & -.0005 & .0000 & -.0001 & .0001 & -.0001 & .0000 & .0000 \\
\hline & $b$ & .0004 & .0000 & .0000 & .0000 & .0001 & .0000 & .0000 & .0000 & .0000 & .0000 & .0000 & .0000 \\
\hline & $c$ & .0668 & -.0057 & -.0006 & -.0020 & .0481 & -.0016 & .0000 & -.0003 & .0193 & -.0004 & .0000 & .0000 \\
\hline & $d$ & .0195 & .0000 & .0000 & .0000 & .0097 & .0000 & .0000 & .0000 & .0049 & .0000 & .0000 & .0000 \\
\hline \multirow{4}{*}{$d$} & $a$ & .0237 & .0010 & .0001 & .0001 & .0116 & .0001 & -.0002 & -.0001 & .0058 & .0001 & .0001 & .0001 \\
\hline & $b$ & .0004 & .0000 & .0000 & .0000 & .0001 & .0000 & .0000 & .0000 & .0000 & .0000 & .0000 & .0000 \\
\hline & $c$ & 1.0233 & .4212 & .3969 & .3911 & 1.0112 & .3907 & .3657 & .3622 & 1.0056 & .3598 & .3344 & .3319 \\
\hline & $d$ & .0194 & .0000 & .0000 & .0000 & .0097 & .0000 & .0000 & .0000 & .0049 & .0000 & .0000 & .0000 \\
\hline \multirow{4}{*}{$e$} & $\bar{a}$ & .0238 & .0005 & -.0003 & -.0004 & .0116 & .0000 & -.0004 & -.0003 & .0057 & .0001 & .0000 & .0000 \\
\hline & $b$ & .0004 & .0000 & .0000 & .0000 & .0001 & .0000 & .0000 & .0000 & .0000 & .0000 & .0000 & .0000 \\
\hline & $c$ & 1.0903 & .0761 & .0194 & .0224 & 1.0501 & .0416 & .0090 & .0096 & 1.0344 & .0240 & .0043 & .0051 \\
\hline & $d$ & .0194 & .0000 & .0000 & .0000 & .0097 & .0000 & .0000 & .0000 & .0049 & .0000 & .0000 & .0000 \\
\hline \multirow{4}{*}{$f$} & $a$ & .0027 & -.0021 & -.0001 & -.0007 & .0006 & -.0008 & .0000 & -.0001 & .0002 & -.0002 & .0001 & .0000 \\
\hline & $b$ & .0004 & .0000 & .0000 & .0000 & .0001 & .0000 & .0000 & .0000 & .0000 & .0000 & .0000 & .0000 \\
\hline & $c$ & .0678 & -.0078 & -.0003 & -.0024 & .0482 & -.0030 & -.0001 & -.0006 & .0193 & -.0009 & .0001 & .0000 \\
\hline & $d$ & .0195 & .0000 & .0000 & .0000 & .0097 & .0000 & .0000 & .0000 & .0049 & .0000 & .0000 & .0000 \\
\hline
\end{tabular}


TABLE 5

MONTE CARLO STANDARD DEVIATION FOR $\tau=1, \rho=0.5,1000$ REPLICATIONS.

\begin{tabular}{|c|c|c|c|c|c|c|c|c|c|c|c|c|c|}
\hline \multirow[b]{2}{*}{$S T$} & \multirow[b]{2}{*}{$D T$} & \multicolumn{4}{|c|}{$n=64$} & \multicolumn{4}{|c|}{$n=128$} & \multicolumn{4}{|c|}{$n=256$} \\
\hline & & $\bar{\nu}$ & $\widetilde{\nu}$ & $\widehat{\nu}_{I}$ & $\widehat{\nu}_{F}$ & $\bar{\nu}$ & $\widetilde{\nu}$ & $\widehat{\nu}_{I}$ & $\widehat{\nu}_{F}$ & $\bar{\nu}$ & $\widetilde{\nu}$ & $\widehat{\nu}_{I}$ & $\widehat{\nu}_{F}$ \\
\hline \multirow{4}{*}{$a$} & $a$ & .0031 & .0069 & .0060 & .0062 & .0011 & .0024 & .0021 & .0022 & .0004 & .0008 & .0007 & .0007 \\
\hline & $b$ & 001 & .0001 & .0001 & .0001 & .0000 & .0000 & .0000 & .0000 & .0000 & .0000 & .0000 & .0000 \\
\hline & $c$ & .0298 & .1120 & .1171 & .1197 & .0160 & .0778 & .0780 & .0793 & .0091 & .0547 & .0492 & .0502 \\
\hline & $d$ & .0001 & .0004 & .0004 & .0004 & .0000 & .0001 & .0001 & .0001 & .0000 & .0000 & .0000 & .0000 \\
\hline \multirow{4}{*}{$b$} & $a$ & .0080 & .0080 & .0071 & .0071 & .0029 & .0025 & .0022 & .0023 & .0012 & .0009 & .0008 & .0008 \\
\hline & $b$ & 001 & .0001 & .0001 & .0001 & .0000 & .0000 & .0000 & .0000 & .0000 & .0000 & .0000 & .0000 \\
\hline & $c$ & .3691 & .0512 & .0462 & .0492 & .2614 & .0223 & .0204 & .0213 & .2163 & .0088 & .0083 & .0085 \\
\hline & $d$ & .0001 & .0004 & .0004 & .0004 & .0000 & .0001 & .0001 & .0001 & .0000 & .0000 & .0000 & .0000 \\
\hline \multirow{4}{*}{$c$} & $a$ & .0132 & .0041 & .0032 & .0033 & .0047 & .0012 & .0009 & .0010 & .0016 & .0002 & .0002 & .0002 \\
\hline & $b$ & .0001 & .0001 & .0001 & .0001 & .0000 & .0000 & .0000 & .0000 & .0000 & .0000 & .0000 & .0000 \\
\hline & $c$ & .5489 & .0100 & .0084 & .0088 & .4008 & .0025 & .0020 & .0020 & .2748 & .0007 & .0005 & .0005 \\
\hline & $d$ & .0014 & .0004 & .0004 & .0004 & .0005 & .0001 & .0001 & .0001 & .0002 & .0000 & .0000 & .0000 \\
\hline \multirow{4}{*}{$d$} & $a$ & .0137 & .0189 & .0168 & .0171 & .0067 & .0087 & .0076 & .0077 & .0031 & .0040 & .0035 & .0035 \\
\hline & $b$ & .0003 & .0003 & .0003 & .0003 & .0001 & .0001 & .0001 & .0001 & .0000 & .0000 & .0000 & .0000 \\
\hline & $c$ & .0269 & .1520 & .1586 & .1658 & .0145 & .1183 & .1241 & .1277 & .0082 & .0922 & .0951 & .0971 \\
\hline & $d$ & .0003 & .0009 & .0008 & .0008 & .0001 & .0002 & .0002 & .0002 & .0000 & .0000 & .0000 & .0000 \\
\hline \multirow{4}{*}{$e$} & $a$ & .0143 & .0214 & .0176 & .0183 & .0065 & .0092 & .0073 & .0075 & .0029 & .0042 & .0034 & .0034 \\
\hline & $b$ & .0003 & .0003 & .0003 & .0003 & .0001 & .0001 & .0001 & .0001 & .0000 & .0000 & .0000 & .0000 \\
\hline & $c$ & .3629 & .0951 & .0855 & .0910 & .2589 & .0550 & .0476 & .0500 & .2148 & .0307 & .0250 & .0269 \\
\hline & $d$ & .0002 & .0009 & .0008 & .0008 & .0001 & .0002 & .0002 & .0002 & .0000 & .0000 & .0000 & .0000 \\
\hline \multirow{4}{*}{$f$} & $a$ & .0153 & .0087 & .0075 & .0076 & .0052 & .0034 & .0026 & .0027 & .0017 & .0009 & .0008 & .0008 \\
\hline & $b$ & .0003 & .0003 & .0002 & .0002 & .0001 & .0001 & .0001 & .0001 & .0000 & .0000 & .0000 & .0000 \\
\hline & $c$ & .5489 & .0189 & .0166 & .0168 & .4005 & .0062 & .0054 & .0055 & .2747 & .0019 & .0016 & .0017 \\
\hline & $d$ & .0014 & .0009 & .0008 & .0008 & .0005 & .0002 & .0002 & .0002 & .0002 & .0001 & .0000 & .0000 \\
\hline
\end{tabular}


TABLE 6

EMPIRICAL SIZE OF $W_{I}, W_{F}$, FOR $\tau=1, \rho=0.5,1000$ REPLICATIONS.

\begin{tabular}{|c|c|c|c|c|c|c|c|c|c|c|c|c|c|}
\hline \multirow[b]{3}{*}{$S T$} & \multirow[b]{3}{*}{$D T$} & \multicolumn{6}{|c|}{$\alpha=.05$} & \multicolumn{6}{|c|}{$\alpha=.10$} \\
\hline & & \multicolumn{2}{|c|}{$n=64$} & \multicolumn{2}{|c|}{$n=128$} & \multicolumn{2}{|c|}{$n=256$} & \multicolumn{2}{|c|}{$n=64$} & \multicolumn{2}{|c|}{$n=128$} & \multicolumn{2}{|c|}{$n=256$} \\
\hline & & $W_{I}$ & $W_{F}$ & $W_{I}$ & $W_{F}$ & $W_{I}$ & $W_{F}$ & $W_{I}$ & $W_{F}$ & $W_{I}$ & $W_{F}$ & $W_{I}$ & $W_{F}$ \\
\hline \multirow{4}{*}{$a$} & $a$ & .065 & .186 & .057 & .134 & .053 & .095 & .113 & .255 & .113 & .191 & .103 & .148 \\
\hline & $b$ & .070 & .182 & .055 & .129 & .053 & .102 & 122 & .252 & .108 & .189 & 108 & 169 \\
\hline & $c$ & .412 & 474 & .472 & .513 & .520 & .535 & 497 & .554 & .572 & .608 & .633 & 646 \\
\hline & $d$ & .068 & .227 & .052 & .130 & .051 & .109 & 113 & .304 & .095 & .201 & 106 & .171 \\
\hline \multirow{4}{*}{$b$} & $a$ & .062 & .194 & .058 & .130 & .057 & .094 & 112 & .267 & .114 & .188 & 102 & .148 \\
\hline & $b$ & .068 & 191 & .052 & .127 & .054 & .096 & 119 & .248 & .113 & .178 & .100 & .164 \\
\hline & $c$ & .060 & 169 & .058 & 139 & .047 & .082 & .121 & .263 & .126 & 199 & .090 & .153 \\
\hline & $d$ & .071 & .228 & .050 & 136 & .058 & .111 & .116 & .297 & .100 & .202 & . 106 & 168 \\
\hline \multirow{4}{*}{$c$} & $a$ & .054 & .199 & .056 & .151 & .052 & .101 & .112 & .272 & .113 & .215 & .096 & .168 \\
\hline & $b$ & .068 & .198 & .059 & .137 & .048 & .087 & .114 & .260 & .112 & .201 & .095 & .158 \\
\hline & $c$ & .077 & .22 & . 044 & .147 & .052 & .120 & .120 & .302 & .101 & .230 & . 102 & 189 \\
\hline & $d$ & .072 & .229 & .045 & 139 & 051 & .111 & .119 & .308 & .096 & .205 & 109 & .168 \\
\hline \multirow{4}{*}{$d$} & $\bar{a}$ & .071 & .191 & .061 & .146 & .053 & .105 & .117 & .254 & .116 & 209 & .108 & .168 \\
\hline & $b$ & .066 & .182 & .064 & 139 & 051 & .099 & .124 & .268 & .105 & .204 & .107 & .167 \\
\hline & $c$ & .889 & .887 & .960 & .958 & .990 & .991 & .925 & .921 & .973 & .972 & .995 & .993 \\
\hline & $d$ & .060 & .219 & .049. & .139. & .047. & . 106 & . 102 & .291 & . 105. & . 197. & .099. & .169 \\
\hline \multirow{4}{*}{$e$} & $\bar{a}$ & .070 & .192 & .054 & .135 & .045 & .101 & .120 & .259 & .114 & .199 & .111 & .161 \\
\hline & $b$ & .069 & . 196. & .059. & .132. & .045. & .102 & . 122 & .265 & . 102. & 194 & .114. & . 175. \\
\hline & $c$ & .094 & .228 & .082. & . 176. & .076. & .137 & . 1755. & .305 & 160 & 242 & .122 & .215 \\
\hline & $d$ & .060 & .221 & .054. & . 136. & .054 & .106 & 109 & .295 & .095. & .195. & 103 & .161 \\
\hline \multirow{4}{*}{$f$} & $\bar{a}$ & .071 & .211 & .058 & .147 & .043 & .096 & .127 & .269 & .105 & .199 & .096 & .162 \\
\hline & $b$ & .072 & .204 & .068 & . 145. & .055. & .112 & . 126. & .268 & . 120. & 213 & .105 & .172 \\
\hline & $c$ & .068 & .235 & .056. & . 144 & .041. & 103 & .124. & .306 & . 114. & 216 & .097 & .165 \\
\hline & $d$ & .057 & .236 & .054 & .144 & .051 & .105 & 113 & .309 & 101 & .199 & .104 & .172 \\
\hline
\end{tabular}


TABLE 7

MONTE CARLO BIAS FOR $\delta=0.6, \gamma=0, D T a, D T b$ and $D T d, \tau=1$, 1000 REPLICATIONS

\begin{tabular}{|c|c|c|c|c|c|c|c|c|c|c|c|c|c|}
\hline \multirow[b]{2}{*}{$\rho$} & \multirow[b]{2}{*}{$D T$} & \multicolumn{4}{|c|}{$n=64$} & \multicolumn{4}{|c|}{$n=128$} & \multicolumn{4}{|c|}{$n=256$} \\
\hline & & $\bar{\nu}$ & $\widetilde{\nu}$ & $\widehat{\nu}_{I}$ & $\widehat{\nu}_{F}$ & $\bar{\nu}$ & $\widetilde{\nu}$ & $\widehat{\nu}_{I}$ & $\widehat{\nu}_{F}$ & $\bar{\nu}$ & $\widetilde{\nu}$ & $\widehat{\nu}_{I}$ & $\widehat{\nu}_{F}$ \\
\hline \multirow[t]{3}{*}{0} & $a$ & .0233 & -.0001 & .0000 & .0000 & .0116 & .0000 & .0000 & .0000 & .0058 & .0000 & .0000 & .0000 \\
\hline & $b$ & .0004 & .0000 & .0000 & .0000 & .0001 & .0000 & .0000 & .0000 & .0000 & .0000 & .0000 & .0000 \\
\hline & $d$ & .0194 & .0000 & .0000 & .0000 & .0097 & .0000 & .0000 & .0000 & .0049 & .0000 & .0000 & .0000 \\
\hline \multirow[t]{3}{*}{0.75} & $a$ & .0237 & .0015 & .0000 & .0000 & .0118 & .0004 & .0000 & .0000 & .0059 & .0001 & .0000 & .0000 \\
\hline & $b$ & .0004 & .0000 & .0000 & .0000 & .0001 & .0000 & .0000 & .0000 & .0000 & .0000 & .0000 & .0000 \\
\hline & $d$ & .0194 & .0000 & .0000 & .0000 & .0097 & .0000 & .0000 & .0000 & .0049 & .0000 & .0000 & .0000 \\
\hline \multirow[t]{3}{*}{-0.5} & $a$ & .0230 & -.0011 & -.0001 & .0000 & .0116 & -.0003 & .0000 & .0000 & .0058 & -.0001 & .0000 & .0000 \\
\hline & $b$ & .0004 & .0000 & .0000 & .0000 & .0001 & .0000 & .0000 & .0000 & .0000 & .0000 & .0000 & .0000 \\
\hline & $d$ & .0194 & .0000 & .0000 & .0000 & .0097 & .0000 & .0000 & .0000 & .0049 & .0000 & .0000 & .0000 \\
\hline
\end{tabular}

TABLE 8

MONTE CARLO SD FOR $\delta=0.6, \gamma=0, D T a, 1000$ REPLICATIONS

\begin{tabular}{|l|l|cccc|cccc|cccc|}
\hline \hline \multirow{2}{*}{$\tau$} & & \multicolumn{9}{|c|}{$n=64$} & \multicolumn{4}{c|}{$n=128$} & \multicolumn{4}{c|}{$n=256$} \\
& $\rho$ & $\bar{\nu}$ & $\widetilde{\nu}$ & $\widehat{\nu}_{I}$ & $\widehat{\nu}_{F}$ & $\bar{\nu}$ & $\widetilde{\nu}$ & $\widehat{\nu}_{I}$ & $\widehat{\nu}_{F}$ & $\bar{\nu}$ & $\widetilde{\nu}$ & $\widehat{\nu}_{I}$ & $\widehat{\nu}_{F}$ \\
\hline \multirow{3}{*}{1} & 0 & .0034 & .0068 & .0068 & .0068 & .0012 & .0024 & .0024 & .0024 & .0004 & .0008 & .0008 & .0008 \\
& 0.75 & .0030 & .0069 & .0048 & .0052 & .0011 & .0024 & .0017 & .0018 & .0004 & .0009 & .0006 & .0006 \\
& -0.5 & .0036 & .0067 & .0060 & .0061 & .0013 & .0023 & .0021 & .0021 & .0005 & .0008 & .0007 & .0007 \\
\hline \multirow{3}{*}{2} & 0 & .0047 & .0096 & .0097 & .0097 & .0017 & .0034 & .0034 & .0034 & .0006 & .0012 & .0012 & .0012 \\
& 0.75 & .0043 & .0097 & .0068 & .0073 & .0016 & .0033 & .0024 & .0025 & .0006 & .0012 & .0008 & .0008 \\
& -0.5 & .0050 & .0094 & .0085 & .0086 & .0018 & .0033 & .0030 & .0030 & .0006 & .0012 & .0010 & .0011 \\
\hline \multirow{3}{*}{0.5} & 0 & .0024 & .0048 & .0048 & .0048 & .0009 & .0017 & .0017 & .0017 & .0003 & .0006 & .0006 & .0006 \\
& 0.75 & .0020 & .0049 & .0034 & .0037 & .0007 & .0017 & .0012 & .0012 & .0003 & .0006 & .0004 & .0004 \\
& -0.5 & .0027 & .0047 & .0042 & .0043 & .0009 & .0016 & .0015 & .0015 & .0003 & .0006 & .0005 & .0005 \\
\hline
\end{tabular}


TABLE 9

EMPIRICAL SIZE OF $W_{I}, W_{F}$ FOR $\delta=0.6, \gamma=0, D T a, D T b$ and $D T d$, $\tau=1,1000$ REPLICATIONS.

\begin{tabular}{|c|c|c|c|c|c|c|c|c|c|c|c|c|c|}
\hline \multirow[b]{3}{*}{$\rho$} & \multirow[b]{3}{*}{$D T$} & \multicolumn{6}{|c|}{$\alpha=.05$} & \multicolumn{6}{|c|}{$\alpha=.10$} \\
\hline & & \multicolumn{2}{|c|}{$n=64$} & \multicolumn{2}{|c|}{$n=128$} & \multicolumn{2}{|c|}{$n=256$} & \multicolumn{2}{|c|}{$n=64$} & \multicolumn{2}{|c|}{$n=128$} & \multicolumn{2}{|c|}{$n=256$} \\
\hline & & $W_{I}$ & $W_{F}$ & $W_{I}$ & $W_{F}$ & $W_{I}$ & $W_{F}$ & $W_{I}$ & $W_{F}$ & $W_{I}$ & $W_{F}$ & $W_{I}$ & $W_{F}$ \\
\hline \multirow{3}{*}{0} & $a$ & .062 & .183 & .061 & .121 & .058 & .099 & .115 & .258 & .110 & .180 & .105 & .159 \\
\hline & $b$ & .062 & .190 & .056 & .121 & .048 & .091 & .114 & .248 & .111 & .172 & .091 & .148 \\
\hline & $d$ & .067 & .218 & .052 & .126 & .057 & .107 & .119 & .305 & .100 & .190 & .107 & .166 \\
\hline \multirow{3}{*}{0.75} & $a$ & .062 & .192 & .059 & .144 & .053 & .111 & .115 & .259 & .110 & .207 & .111 & .163 \\
\hline & $b$ & .064 & .179 & .056 & .124 & .055 & .104 & .121 & .245 & .106 & .197 & .117 & .163 \\
\hline & $d$ & .064 & .225 & .044 & .136 & .047 & .111 & .115 & .310 & .094 & .204 & .101 & .181 \\
\hline \multirow{3}{*}{-0.5} & $a$ & .060 & .196 & .058 & .122 & .055 & .103 & .100 & .258 & .109 & .193 & .097 & .158 \\
\hline & $b$ & .055 & .171 & .046 & .111 & .046 & .089 & .101 & .241 & .089 & .178 & .088 & .149 \\
\hline & $d$ & .068 & .245 & .057 & .143 & .058 & .114 & .128 & .312 & .101 & .196 & .104 & .167 \\
\hline
\end{tabular}

Table 10 Empirical analysis of the PPP:

semiparametric analysis of fractional cointegration.

\begin{tabular}{cccccccccc}
\hline & $\widetilde{\delta}_{y}$ & $\widetilde{\delta}_{x}$ & $\widetilde{\delta}_{*}$ & $\left(\widetilde{T}_{y x}\right)^{2}$ & $\left(\bar{T}_{y x}\right)^{2}$ & $\left(\bar{H}_{y}\right)^{2}$ & $\left(\bar{H}_{x}\right)^{2}$ & $\bar{\nu}$ & $\widetilde{\gamma}$ \\
\hline \hline Ph-NY & 1.40 & 1.53 & 1.35 & 4.83 & 0.56 & 0.26 & 4.00 & 0.99 & 0.82 \\
Bo-NY & 1.45 & 1.53 & 1.38 & 1.28 & 0.18 & 2.83 & 0.68 & 1.02 & 1.05 \\
Ph-Bo & 1.40 & 1.45 & 1.21 & 0.67 & 0.10 & 4.53 & 7.66 & 0.97 & 0.83
\end{tabular}

Note: critical values for $\chi_{1}^{2}: 3.84(5 \%)$ and $6.63(1 \%)$.

Table 11 Empirical analysis of the PPP: parametric analysis of fractional cointegration.

\begin{tabular}{ccccccccc}
\hline & $L R_{B}$ & $\widehat{\delta}$ & $\widehat{\gamma}$ & $L R_{\{\delta=1, \gamma=0\}}$ & $L R_{\{\gamma=1\}}$ & $\widehat{\nu}$ & $\widehat{\mu}_{21}$ & $L R_{\{\nu=1\}}$ \\
\hline \hline Ph-NY & $9.69[0.287]$ & 1.42 & 0.66 & $21.50[0.000]$ & $9.83[0.002]$ & 0.98 & 0.0045 & $3.74[0.053]$ \\
Bo-NY & $4.51[0.808]$ & 1.43 & 0.69 & $35.87[0.000]$ & $9.85[0.002]$ & 1.02 & 0.0041 & $2.21[0.137]$
\end{tabular}

Note: $L R_{B}$ is the likelihood ratio statistic for the hypothesis that the second and third lag are excluded; $L R_{\{\delta=1, \gamma=0\}}, L R_{\{\gamma=1\}}, L R_{\{\nu=1\}}$ are the the likelihood ratio statistics for the hypothesis $H_{0}:\{\delta=1, \gamma=0\}, H_{0}:\{\gamma=1\}$, $H_{0}:\{\nu=1\}$; P-values are in brackets. 
Figure 1 Normalised log prices, New York, Philadelphia, Boston;

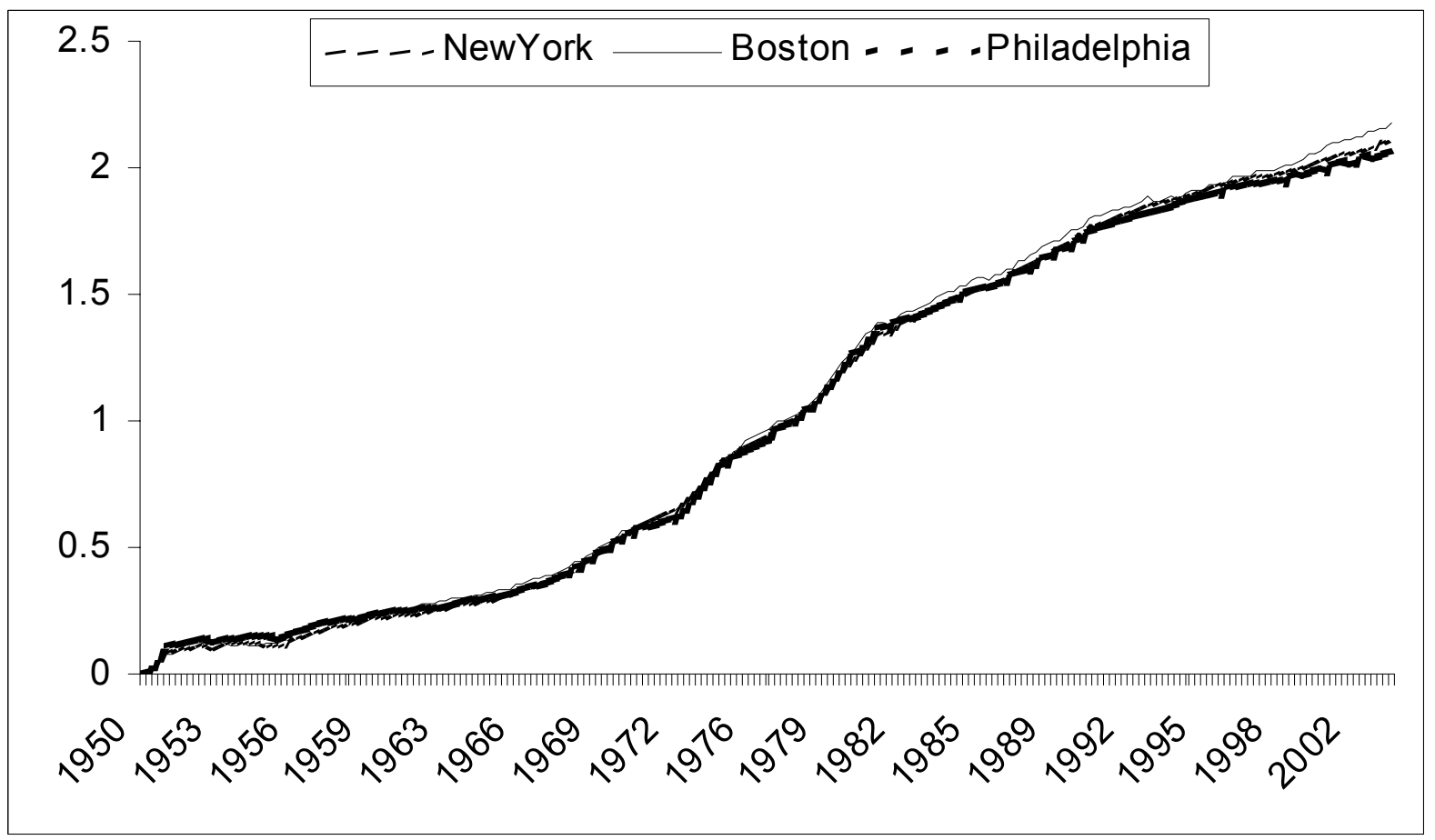

Figure 2 Inflation rate, New York, Philadelphia, Boston;

(rescaled first differences of normalised log prices).

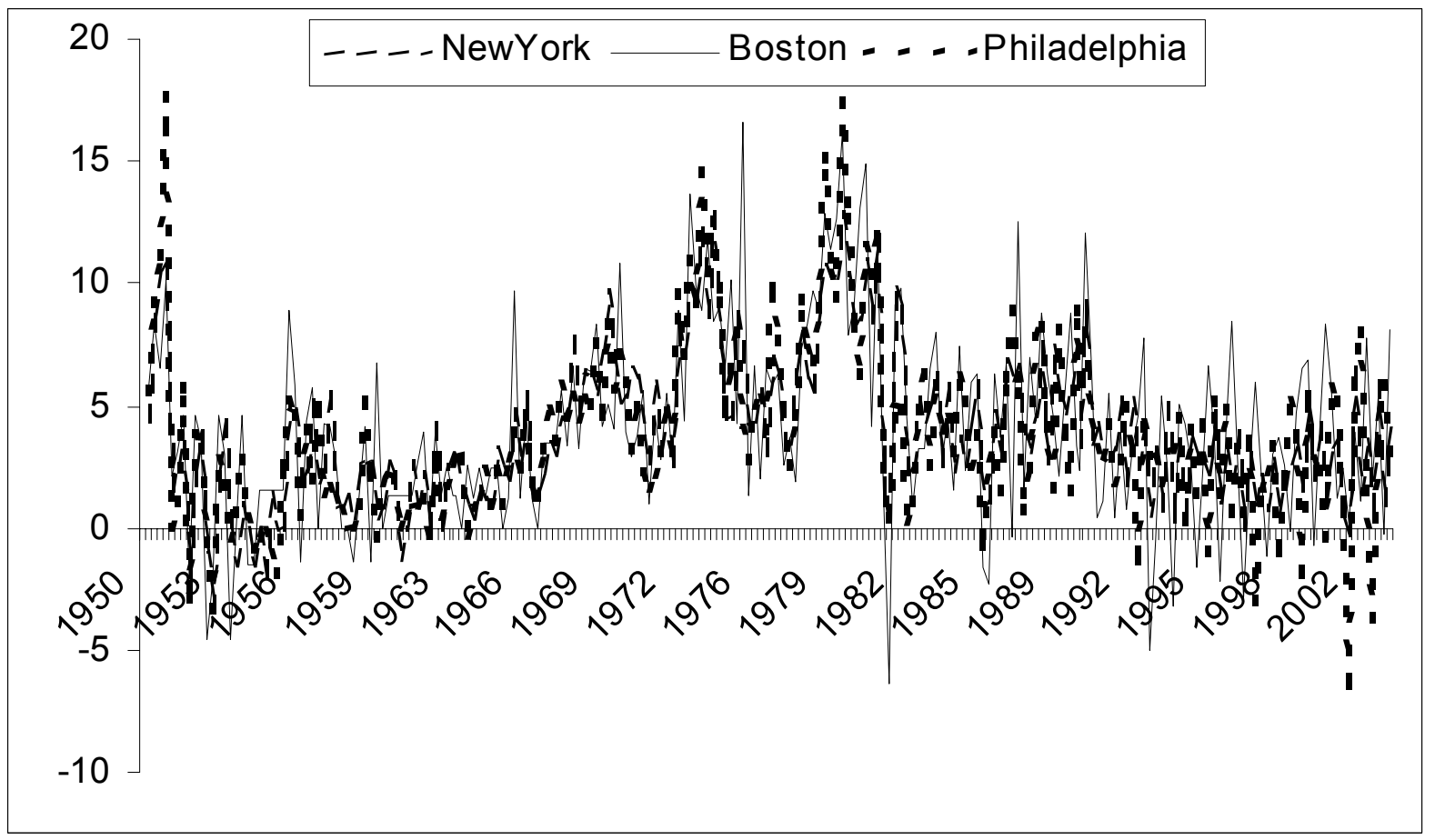


Figure 3 GLS cointegrating residuals $y_{t}-\widehat{v} x_{t}$

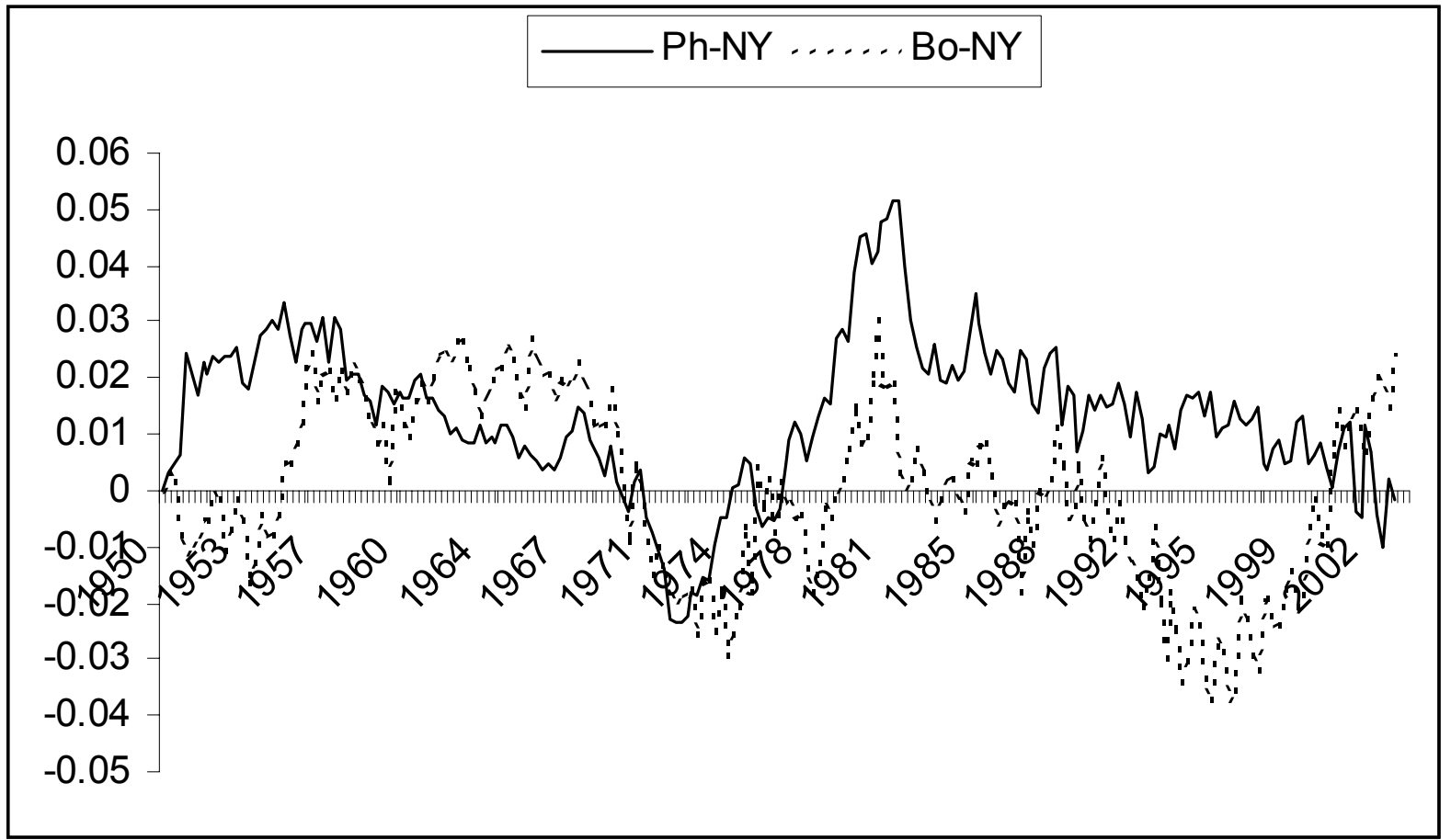

Figure 4 Restricted cointegrating residuals $y_{t}-x_{t}$

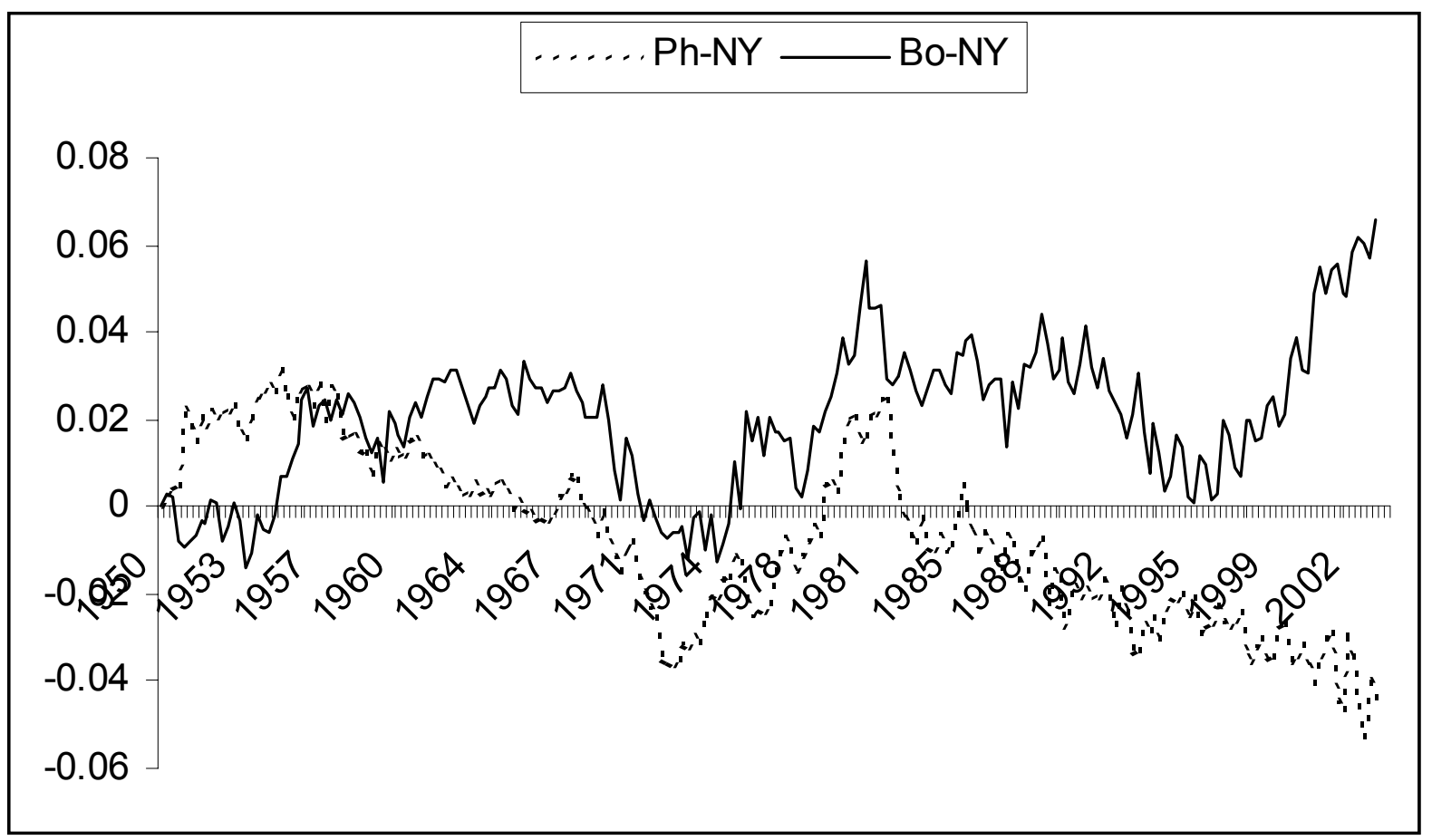

